\title{
Identification of Novel Survival Related IncRNA- miRNA-mRNA Competing Endogenous RNA Network Associated with Immune Infiltration in Colorectal Cancer
}

\section{Jianxin Li}

The Affiliated Hospital of Southwest Medical University https://orcid.org/0000-0001-9863-6493

\section{Ting Han}

The Affiliated Hospital of Southwest Medical University

\section{Xin Wang}

The Affiliated Hospital of Southwest Medical University

\section{Yinchun Wang}

The Affiliated Hospital of Southwest Medical University

Qingqiang Yang ( $\square$ yangqingqiang121@163.com)

The Affiliated Hospital of Southwest Medical University https://orcid.org/0000-0001-7304-3501

\section{Primary research}

Keywords: Colorectal Cancer, Competing Endogenous RNA, Long Noncoding RNA, MicroRNA, Bioinformatic Analysis, Immune Infiltration

Posted Date: November 25th, 2020

DOl: https://doi.org/10.21203/rs.3.rs-113476/v1

License: (c) (i) This work is licensed under a Creative Commons Attribution 4.0 International License. Read Full License 


\section{Abstract}

Background: Increasing studies have reported that long noncoding RNAs (IncRNAs) play critical roles in the initiation and progression of carcinogenesis. However, the underlying regulatory mechanisms of IncRNA related competing endogenous RNA (ceRNA) network in colorectal cancer (CRC) are not fully understood.

Methods: Dysregulated microRNAs (miRNAs) in CRC samples were screened from the Gene Expression Omnibus (GEO) and The Cancer Genome Atlas (TCGA) database. After that, the key miRNAs were filtered out through a comprehensive assessment of their expression levels and prognostic values. Subsequently, the targeted downstream mRNAs and upstream IncRNAs of the key miRNAs were predicted by using multiple bioinformatic databases. A ceRNA network was constructed by using Cytoscape, and the Gene Ontology (GO) annotation and Kyoto Encyclopedia of Genes and Genomes (KEGG) pathway analyses were performed on this network using the DAVID database. Ultimately, expression levels and prognostic values of the IncRNAs and mRNAs were evaluated, and a survival related ceRNA network was constructed and visualized by using Cytoscape. In addition, the Gene Set Enrichment Analysis (GSEA) software package was employed to identify the pathways in which this survival related ceRNA network was enriched. Furthermore, correlations of ceRNA network with immune infiltration level were estimated by the Tumor Immune Estimation Resource (TIMER) databases.

Results: In total, 28 dysregulated miRNAs were obtained, and two of them were identified as key miRNAs based on expression levels and prognostic values analyses. Subsequently, a total of three upstream IncRNAs and 309 downstream mRNAs were predicted by using bioinformatic tools, and two key IncRNAs and eight key mRNAs were identified by expression and survival analysis. A ceRNA regulatory network associated with the prognosis of CRC patients was constructed. Furthermore, GSEA analysis indicated the possible association of key mRNAs with CRC onset and progression. Importantly, immune infiltration analysis revealed that the ceRNA network was remarkably associated with infiltration abundance of multiple immune cells and expression levels of immune checkpoints.

Conclusions: We constructed a survival related ceRNA regulatory network in human CRC, NEAT1 and XIST are potential prognostic factors that affect CRC onset and progression by targeting miR-195-5p.

\section{Introduction}

Malignant tumor, with more than 18.0 million newly diagnosed cases and 9.0 million deaths in 2018 , has become the main threatening public health problem around the world [1]. Among them, colorectal cancer (CRC) is one of the most frequently diagnosed digestive malignancies, which remains the third leading cause of cancer related death worldwide [1][2]. Early diagnosis and timely radical resection are critical for CRC. The 5-year survival rate is approximately $90 \%$ when the localized disease is diagnosed at an early stage. However, the 5-year survival rate of patients with distant metastasis dropped sharply to only $12 \%$ [3]. Metastasis and recurrence are the major causes of death in human CRC. It is estimated that more 
than $50 \%$ of CRC patients will die from metastasis-related complications, which might be due to the lack of specific and sensitive biomarkers [4]. To make matters worse, available epidemiological data indicate that the incidence rates of human CRC have increased at a faster pace than any other types of cancer around the world [5]. Despite advances in surgical technology and targeted therapy, the prognosis of patients with $\mathrm{CRC}$ remains dismal because of recurrence and distant metastasis. Therefore, exploring the molecular mechanisms underlying colorectal carcinogenesis is imperative and meaningful to provide novel prognostic and therapeutic biomarkers for clinical CRC treatment.

The occurrence of CRC is a complex multi-stage process involving accumulation of various genetic and epigenetic alternations [6]. Increasing evidence in recent years have demonstrated that long noncoding RNA (IncRNA) plays a significant role in the development of multiple types of malignant tumor, including CRC [7-10]. LncRNA is a class of noncoding transcripts with transcript more than 200 nucleotides in length [11]. Salmena et al [12] first proposed the competing endogenous RNA (ceRNA) hypothesis that genes could achieve crosstalk among each other by forming a regulatory network. The ceRNA hypothesis postulates that IncRNAs could act as endogenous miRNA sponges and inhibit miRNA function, and thereby modulating miRNA-mediated target inhibition. In other words, the expression level of miRNA could be negatively correlated with its upstream IncRNA and downstream mRNA simultaneously [13]. Therefore, ceRNA crosstalk is a vital mechanism underlying the complex pathogenesis and multistage progression of human CRC, but our knowledge about the IncRNA related ceRNA network in human CRC remains limited, exploring the ceRNA regulatory network might be a potential pointcut for identifying novel early diagnostic and therapeutic targets of CRC and deserves further research.

In the present study, dysregulated miRNAs in human CRC were identified by mining the gene expression profiles obtained from the Gene Expression Omnibus (GEO) and The Cancer Genome Atlas (TCGA) database. Subsequently, two miRNAs were identified as key miRNA for further study through comprehensive consideration of the expression levels and prognostic values of these miRNAs in human $\mathrm{CRC}$. Then, the upstream IncRNAs and downstream mRNAs of the key miRNAs were predicted by using multiple bioinformatic tools, and a ceRNA regulatory network was constructed and visualized by using Cytoscape software. In addition, the Gene Ontology (GO) annotation and Kyoto Encyclopedia of Genes and Genomes (KEGG) pathway enrichment analyses of the ceRNA regulatory network were perform by using the DAVID database. Ultimately, the expression levels and prognostic values of the upstream IncRNAs and downstream mRNAs in the ceRNA regulatory network were assessed and a novel ceRNA network in which each RNA are significantly correlated with prognosis of patients with CRC was constructed successfully. Furthermore, we used Gene Set Enrichment Analysis (GSEA) software package to explore the pathway enrichment of this survival related ceRNA regulatory network. In addition, Tumor Immune Estimation Resource (TIMER) database was used to estimate the correlations between ceRNA network and immune infiltration level in CRC microenvironment. Through our efforts, it is hoped that the results of our research will contribute to enhance our understanding of the regulatory mechanisms underlying the pathogenesis of human CRC and find new therapeutics for clinical CRC treatment. 


\section{Materials And Methods}

\subsection{Data download and preprocessing.}

To screen out the dysregulated miRNAs in human CRC, human miRNA expression profiles GSE108153 (including 21 cancer samples and 21 adjacent normal samples) [14] was downloaded from the Gene Expression Omnibus database (GEO, http://www.ncbi.nlm.nih.gov/geo) [15], which was based on the GPL19730 platform (Agilent-046064 Unrestricted_Human_miRNA_V19.0_Microarray). Meanwhile, to enhance the reliability of our results, miRNA expression profiles (including 9 normal samples and 539 cancer samples) and clinical information (including 547 cases) of patients with CRC were obtained from The Cancer Genome Atlas (TCGA, https://gdc-portal.nci.nih.gov/) database.

\subsection{Identification of dysregulated miRNAs.}

Firstly, we converted the miRNA probe IDs in the matrix files to mature miRNA name based on the annotation document of its platform files through the Perl language (https://www.perl.org/).

Subsequently, the "Limma" package in R software (V 3.6.2; http://www.r-project.org/) was used to identify differentially expressed miRNAs between CRC samples and normal samples both in miRNA expression profiles obtained from GEO and TCGA databases, adjusted $P$-value of $<0.05$ and absolute value of log2 fold change (log2 FC) $\geq 1$ were considered as the cut-off criteria [16]. Finally, the differentially expressed miRNAs dysregulated in both GEO and TCGA datasets were chosen for subsequent analysis by using the intersect function of Venn Diagram (http://bioinformatics.psb.ugent.be/webtools/Venn/).

\subsection{Identification of key miRNAs.}

To identify the key miRNAs associated with prognosis of CRC patients, the expression levels and prognostic values of the dysregulated miRNAs were assessed by using clinical information obtained from TCGA database. The dysregulated miRNAs not only differentially expressed between the CRC cancer samples and normal samples, but also associated with the prognosis of CRC patients were identified as key miRNAs in human CRC and were chosen for subsequent research, according to the criteria of a $P$ value $<0.05$. In addition, we further validated the expression pattern of key miRNAs in multiple types of cancer by using the Gene Expression Display Server (GEDS, http://bioinfo.life.hust.edu.cn/web/GEDS/) database, a web-based tool for quantification, comparison and visualization of gene expression data based on The Cancer Genome Atlas, Genotype-Tissue Expression (GETx), Cancer Cell Line Encyclopedia (CCLE), and MD Anderson Cell Lines Project (MCLP) databases [17].

2.4 Construction of ceRNA regulatory network and functional enrichment analysis

The starBase database (http://starbase.sysu.edu.cn/) is an online tool which allows researchers performing Pan-Cancer analysis on RNA-RNA and RBP-RNA interactions [18]. The LncBase V2 database (http://carolina.imis.athena-innovation.gr/) is a web-based tool which caters information regarding cell type specific miRNA-IncRNA interaction and enables users to easily identify interactions in 66 different cell types, spanning 36 tissues for human and mouse [19]. The upstream IncRNAs which interacted with 
the key miRNAs were predicted by using these two databases, only those IncRNAs predicted by both two databases and interacted with all key miRNAs simultaneously were identified as upstream IncRNAs. Subsequently, the downstream mRNAs which interacted with the key miRNAs were predicted by using miRTarBase (http://mirtarbase.mbc.nctu.edu.tw/) [20], targetScan (http://www.targetscan.org) [21] and miRDB (http://www.mirdb.org/) [22]. To enhance the reliability of the predicted interaction relationships, only the predicted mRNAs presented in all three databases were identified as the downstream mRNAs. Ultimately, the IncRNA-miRNA-mRNA regulatory network was constructed and visualized by Cytoscape software (v3.7.2, http://www.cytoscape.org/) [23]. Furthermore, the functional enrichment analysis of this ceRNA regulatory was performed by using the Database for Annotation, Visualization and Integrated Discovery (DAVID v6.8 https://david.ncifcrf.gov/home.jsp) [24] bioinformatics database, only the terms with $P$-value of $<0.05$ was considered as statistically significant.

2.5 Gene expression validation and survival analysis.

To further enhance the reliability of the predicted interaction relationships, the expression levels of upstream IncRNAs were assessed by using the Gene Expression Profiling Interactive Analysis (GEPIA; http://gepia.cancer-pku.cn/detail.php), a web-based tool which provides fast and customizable functionalities including differential expression analysis, profiling plotting, correlation analysis, and patient survival analysis based on TCGA and Genotype-Tissue Expression data [25]. The expression levels of downstream mRNAs were assessed by using the gene expression profiles in human CRC obtained from TCGA database. Subsequently, the prognostic values of the upstream IncRNAS and downstream mRNAs were validated in human CRC by using the PrognoScan (http://dna00.bio.kyutech.ac.jp/PrognoScan/) database, a web-based tool for assessing the biological relationship between gene expression and prognosis of multiple types of cancer [26]. Only genes complied with the ceRNA hypothesis and associated with the prognosis of patients with CRC were chosen for subsequent analysis.

2.6 Identification of survival related ceRNA regulatory network and functional annotation.

Based on the prediction and evaluation above, a novel IncRNA-miRNA-mRNA regulatory network associated with the prognosis of CRC patients was constructed and visualized by Cytoscape software. In addition, to further explore the underlying role of this survival related ceRNA regulatory network in the process of human CRC, functional annotation of genes in the ceRNA regulatory network was analyzed by the Gene Set Enrichment Analysis (GSEA, https://www.gsea-msigdb.org/) database, an initial database of 1,325 biologically defined gene sets [27]. The enriched terms with $P$-value of $<0.05$ were considered statistically significant.

2.7 Correlation of ceRNA network with immune infiltration level in human CRC.

The Tumor Immune Estimation Resource (TIMER, https://cistrome.shinyapps.io/timer/) is an online database which established for systematical analysis of immune infiltrates across multiple cancer types [28]. We applied the TIMER database to assess the correlations of gene expression in the ceRNA 
regulatory network with the level of immune infiltrates in human CRC. In addition, the correlation between the ceRNA regulatory network and abundance of T-cell infiltration in human CRC were confirmed by using the GEPIA database.

\subsection{Statistical analysis}

Most of the statistical analyses were carried out through the bioinformatic tools mentioned above, while the rest were carried out through $\mathrm{R}$ software ( $\mathrm{v}$ 3.6.2). Identification of differentially expressed genes were assessed by Student's t-test, Benjamini-Hochberg False Discovery Rate (FDR) method was applied to adjust the $P$ value. Kaplan-Meier curve analysis with log-rank test was used to assess the prognostic values of miRNAs in human CRC. Spearman correlation coefficients were used to assess the correlations. A $P$-value $<0.05$ was considered statistically different.

\section{Results}

3.1 Identification of dysregulated miRNAs in human CRC.

As shown in the volcano plot and heatmap, differentially expressed miRNAs in GEO and TCGA datasets were identified separately with a threshold predefined (Fig. 1A-D). In the GSE108153 dataset, a total of 49 dysregulated miRNAs were identified. Meanwhile, a total of 504 dysregulated miRNAs were also screened out in the TCGA dataset. A total of 28 dysregulated miRNAs were selected for subsequent analyses by using the intersect function of Venn Diagram (Fig. 1E).

\subsection{Identification of key miRNAs.}

In order to evaluate the prognostic values of dysregulated miRNAs for overall survival in human CRC, clinical information including survival status and survival time of 547 patients with CRC were obtained from the TCGA database. The results showed that five of the dysregulated miRNAs (miR-195-5p, miR-10b$5 p$, miR-21-3p, miR-125b-5p, and miR-145-5p) were significantly associated with prognosis of patients with CRC ( $P<0.05$; Fig. 2A-E). In the meanwhile, we compared the expression levels of these survival related miRNAs between cancer samples and normal samples in TCGA dataset. After combination of the prognostic value and expression level, we found that only two of them (miR-195-5p and miR-10b-5p) showed both high expression and poor prognosis in human CRC $(P<0.05 ;$ Fig. $2 \mathrm{~F}-\mathrm{J})$. To further enhance the reliability of our screening results, we verified the two dysregulated miRNAs in the GEDS database. As shown in Fig. $2 \mathrm{~K}$ and $\mathrm{L}$, miR-195-5p and miR-10b-5p were dysregulated in multiple cancer types including CRC. Subsequently, those two miRNAs were chosen for next analyses.

\subsection{Construction of ceRNA regulatory network.}

The upstream IncRNAs that could potentially bind to the key miRNAs were predicted through starBase and LncBase V2 databases, a total of three upstream IncRNAs were predicted to regulate the two key miRNAs simultaneously (Fig. 3A). Subsequently, in order to identify downstream mRNAs that could potentially be targeted by those two key miRNAs, we applied miRTarBase, targetScan and miRDB to 
improve the credibility of the predicted results. As a result, a total of 287 downstream mRNAs were predicted to be targeted by miR-195-5p, while there were 22 downstream mRNAs that could potentially be targeted by miR-10b-5p (Fig. 3B \& C). Based on the prediction above, a ceRNA regulatory network comprised of six IncRNA-miRNA pairs and 310 miRNA-mRNA pairs was established and visualized by Cytoscape software (Fig. 3D).

\subsection{Functional analysis for the ceRNA regulatory network}

We used DAVID database to further explore the biological functions of the ceRNA regulatory network. A total of $222 \mathrm{GO}$ terms and $45 \mathrm{KEGG}$ pathways were enriched, and the top 10 enriched G0 terms of each group and the top 20 enriched KEGG pathways according to $P$ value were displayed (Tables $1 \& 2$ ). In the biological processes (BP) group, the ceRNA regulatory network mainly enriched in the process of regulation of transcription from RNA polymerase II promoter, regulation of cell migration, regulation of cell adhesion, and regulation of apoptotic process. In the cellular component (CC) group, the ceRNA regulatory network mainly enriched in nucleoplasm, cytoplasm, and nucleus. In the molecular function (MF) group, the ceRNA regulatory network mainly enriched in protein binding, beta-catenin binding, and ubiquitin protein ligase activity. These result above indicated that the ceRNA network was significantly enriched in terms correlated with cell proliferation, cell migration, and cell apoptosis. The KEGG enrichment analysis revealed that the ceRNA regulatory network was significantly enriched in multiple cancer related pathways such as PI3K-Akt signaling pathway, FoxO signaling pathway, Signaling pathways regulating pluripotency of stem cells, and AMPK signaling pathway.

\subsection{Identification and validation of key IncRNA and mRNA.}

Based on the ceRNA hypothesis, the miRNA could be negatively correlated with its upstream IncRNA and downstream mRNA simultaneously. Therefore, we assessed the expression levels and prognostic values of the predicted IncRNAs for overall survival in patients with CRC by using GEPIA and PrognoScan databases, respectively. Only two IncRNAs (NEAT1 and XIST) showed both low expression and improved prognosis in patients with CRC (Fig. 4A \& B). As for downstream mRNA, we collected gene expression profiles in human CRC from TCGA database to assess the expression levels of the predicted mRNA. In the meanwhile, the prognostic values of those mRNAs for overall survival in patients with CRC were also assessed using the PrognoScan database. A total of 1964 downregulated genes were identified in human CRC from TCGA database, we then integrated the 1964 downregulated genes with the downstream mRNAs in the ceRNA regulatory network. As a result, no specific gene was identified to be targeted by miR-10b-5p, while there were 16 specific genes that could potentially be targeted by miR-195-5p (Fig. 4C \& D). Subsequently, survival analysis found that eight of the 16 specific genes (ACOX1, CYP26B1, IRF4, ITPR1, LITAF, PHLPP2, RECK, and TPM2) were significantly associated with prognosis of CRC patients. The expression boxplot and survival curve of those mRNAs were displayed in Fig. 4E-L. Those two IncRNAs and eight mRNAs were chosen for further analysis.

3.6 Integration of survival related ceRNA regulatory network and GSEA enrichment analysis. 
Based on the validation above, a survival related ceRNA regulatory network including two IncRNA-miRNA pairs (NEATI-miR-195-5p and XIST-miR-195-5p) and eight miRNA-mRNA pairs (miR-195-5p-

ACOX1/CYP26B1/IRF4/ITPR1/LITAF/PHLPP2/RECK/TPM2) was established (Fig. 5A). Each component in the ceRNA regulatory network had significant prognostic values in human $\mathrm{CRC}$, and fully compliance with the rule of ceRNA hypothesis. After that, we used GSEA software package to further explore the biological function of the survival related ceRNA regulatory network. As shown in Fig. 5B, GSEA enrichment analysis indicated that the survival related ceRNA regulatory network was significantly enriched in multiple cancer related pathways, such as JAK-STAT signaling pathway, mTOR signaling pathway, TGF- $\beta$ signaling pathway, Wnt signaling pathway, ERBB signaling pathway, and VEGF signaling pathway.

\subsection{Correlation of ceRNA network with immune infiltration level in human CRC.}

The correlation between the survival related ceRNA regulatory network and tumor-infiltrating immune cells in the CRC microenvironment was assessed by using TIMER. As shown in Fig. 6, We found that genes expression in the ceRNA regulatory network was significantly associated with infiltrating immune cells, including B cells, $\mathrm{CD} 4^{+} / \mathrm{CD} 8^{+} \mathrm{T}$ cells, Macrophages, Neutrophils, and Dendritic cells, in human CRC microenvironment. Subsequently, the correlation between the ceRNA regulatory network and abundance of $T$ cells infiltration in human CRC were further evaluated by using the GEPIA database, which indicated that the survival related ceRNA regulatory network is closely associated with abundance of Central memory T cells $(r=0.75, P=0)$, Effector T cells $(r=0.73, P=0)$, Effector Treg T-cells $(r=0.74, P=0)$, Exhausted T cells $(r=0.68, P=0)$, Naïve T cells $(r=0.72, P=0)$, Resident memory T cells $(r=0.69, P=0)$, Resting Treg T-cells $\left(r=0.76, P=3.9 \mathrm{e}^{-69}\right)$, and Th1-like $(r=0.72, P=0)($ Fig. 7A-H). We also found that the ceRNA regulatory network is closely associated with the expression of multiple well-known immune checkpoints, such as PDL1 $\left(r=0.48, P=1.6 \mathrm{e}^{-16}\right)$, CTLA-4 $\left(r=0.60, P=1.3 \mathrm{e}^{-37}\right), \mathrm{TIM}-3\left(\mathrm{r}=0.71, P=1.1 \mathrm{e}^{-}\right.$ $\left.{ }^{56}\right)$, and LAG-3( $\left(r=0.46, P=2 \mathrm{e}^{-20}\right)$, suggesting that the ceRNA regulatory network participates in the process of immune checkpoint mediated T cell failure (Fig. 7l-L). Therefore, these findings further support that this survival related ceRNA regulatory network was closely correlated with immune infiltration level in human CRC, suggesting that the ceRNA regulatory network might be important participant in immune escape in the CRC microenvironment.

\section{Discussion}

Colorectal cancer is a main threatening public health problem worldwide with its high morbidity and mortality in both men and women. Although numerous studies have been launched to explore the pathogenesis of CRC and to improve the clinical outcome of patients with CRC, it still remains the third leading cause of cancer related death worldwide [1]. Therefore, to explore the mechanisms underlying the pathogenesis and progression of human CRC and identify potential therapeutic targets for clinical treatment of CRC is meaningful and imperative. Recently, the dysregulated IncRNA related ceRNA regulatory network has been widely reported to be involved in carcinogenesis of multiple cancer types, including CRC. For instance, IncRNA CRNDE has been reported to function as a sponge for miR-181a-5p, 
thereby promoting the progression and chemoresistance of CRC cells through modulating the expression levels of TCF4 and the activity of Wnt/ $\beta$-catenin signaling [29]. Previous research reported that IncRNA SNHG1 promotes CRC cell growth by sponging miR-154-5p to regulate the expression level of CCND2, which plays critical role in cell cycle progression [30]. LncRNA MIR17HG was reported to increase the expression level of NF-KB/RELA through competitively sponging miR-375, thereby promoting tumorigenesis and metastasis in CRC cells. In addition, MIR17HG was reported to upregulate the expression of PD-L1, suggesting that it may participant in immune escape in the CRC microenvironment [31]. Nevertheless, the clinical utility of IncRNA related ceRNA regulatory network is limited at present, exploring the IncRNA related ceRNA regulatory network will help to provide a comprehensive perspective of the molecular mechanisms of gene interaction and regulation in human CRC.

In the present study, dysregulated miRNAs with prognostic significance in human CRC were identified in cancer samples compared with adjacent non-cancer samples from TCGA and GEO databases. Then, a novel IncRNA-miRNA-mRNA regulatory network involved in CRC was constructed through stepwise prediction and validation. Functional enrichment analysis revealed that this novel ceRNA regulatory network was significantly enriched in some cancer related pathways, such as PI3K-Akt signaling pathway [32], FoxO signaling pathway [33], Wnt signaling pathway [34], and AMPK signaling pathway [35]. To enhance the reliability of our study, we further assessed the expression levels and prognostic values of genes in the ceRNA regulatory network and constructed a survival related ceRNA regulatory network, each constituent in the network has prognostic significance in human CRC, which might provide novel diagnostic and therapeutic targets for clinical treatment of CRC.

LncRNAs are defined as transcripts that are $>200$ nucleotides in length and have no ability to code protein [36]. Previous studies have reported that IncRNA participate in multiple biological processes and tumorigenesis by sponging miRNA [37]. In the present study, we found that IncRNA NEAT1 and XIST not only dysregulated in CRC, but also significantly associated with poor prognosis, and these two IncRNAs were identified as key IncRNA in CRC. Studies have shown that expression of IncRNA XIST was dysregulated in CRC cell lines and tissues. XIST knockdown inhibited the cell migration and invasion of CRC cells in vitro and in vivo by regulating the processes of epithelial-mesenchymal transition (EMT), an important step in tumor progression and metastasis [38][39]. In addition, dysregulated XIST has been found to be involved in chemoresistance of CRC to Doxorubicin, with the silencing of XIST significantly enhancing the anti-tumor effect of Doxorubicin in CRC [40]. There is also increasing evidence that IncRNA NEAT1 was dysregulated in human CRC tissues and was significantly correlated with a worse overall survival in CRC patients. NEAT1 regulates the Wnt/ $\beta$-catenin signaling pathway by targeting DDX5, thereby facilitating CRC cells migration and invasion in vitro and in vivo [41]. Furthermore, NEAT1 also has been found to participate in chemoresistance of CRC, NEAT1 knockdown increased the sensitivity of 5-fluorouracil and promoted apoptosis in CRC cells [42]. These studies above improved the reliability of our research that dysregulation of IncRNA XIST and NEAT1 was closely correlated with the pathogenesis of CRC, which is valuable for further study. 
MiRNAs are defined as endogenous non-coding RNAs that are 21-25 nucleotides in length and suppress protein coding gene expression by binding to the 3 '-untranslated regions (UTRs) of mRNAs [43].

Dysregulation of miRNAs have been reported to be associated with the onset and progression of human CRC. For instance, expression of miR-330 was downregulated in CRC samples compared with normal control, CRC patients with low miR-330 expression showed poorer overall survival than those with high miR-330 expression. Mechaismly, miR-330 suppresses cell proliferation, migration, invasion, and angiogenesis by reducing the phosphorylation of AKT and STAT3 via targeting HMGA2 in vitro [44]. Moreover, expression of miR-128-3p in tumor samples was closely associated with oxaliplatin sensitivity in human CRC, forced-expression of miR-128-3p significantly improved the sensitivity of CRC cells to oxaliplatin [45]. In the present study, we found that miR-195-5p was sponged by XIST and NEAT1, and was significantly associated with the prognosis of patients with CRC, miR-195-5p was identified as a potential biomarker in CRC. Studies have shown that expression of miR-195-5p was dysregulated in CRC, and miR-195-5p acts as an independent risk factor for overall survival in CRC [46]. Importantly, miR-195$5 p$ has been proved to regulate the stemness and chemoresistance of CRC cells through inhibiting Notch signaling pathway, forced-expression of miR-195-5p significantly improved the sensitivity of CRC cells to 5-fluorouracil [47]. Intriguingly, we also found that the role of NEAT1/XIST-miR-195-5p regulatory axes in human cancer have been proved by previously studies, which partially enhanced the reliability of our results [48][49]. However, studies focused on the significance of NEAT1/XIST-miR-195-5p regulatory axes in $\mathrm{CRC}$ remains largely limited, they are worthy of further explore.

We further analyzed and proved the downstream mRNAs that targeted by the key miRNA, and eight survival related mRNAs were identified as key mRNAs in CRC that regulated by miR-195-5p. In agreement with our results, previous studies have reported that these key mRNAs participate in the initiation and progression of multiple types of cancer, including CRC. IRF4, as a member of the interferon regulating factor family, is downregulated by PIP5K1A in human CRC [50]. PHLPP2 belongs to the phosphokinase family that has been found to be down-expressed in CRC, and CRC patients with high PHLPP2 expression showed better prognosis than those with low PHLPP2 expression [51]. TPM2 has been found to be downregulated in CRC cell lines and tissues, and reduction of TPM2 was associated with increased cell migration and cell proliferation of CRC cells in vitro [52]. RECK is a unique matrix metalloproteinase (MMP) regulator that has been found to be lowly expressed in CRC tissues and cell lines. RECK knockdown enhanced the migratory and invasive rates of CRC cells [53]. ITPR1 is an isoforms of inositol 1,4,5-trisphosphate receptor (ITPR), and there is increasing evidence that ITPR expression was associated with aggressiveness of CRC cells [54]. CYP26B1 belongs to the CYP26 enzymes hydroxylate retinoic acid family that has been reported to be dysregulated in CRC, and CYP26B1 expression was significantly correlated with clinical outcome of patients with CRC [55]. ACOX1, which catalyzes the initial step for peroxisomal $\beta$-oxidation, has been indicated to associated with peroxisomal disorders and carcinogenesis in the liver cancer [56]. LITAF has been reported to function as a tumor suppressor and is frequently down-expressed in multiple cancer types because its expression is regulated by the tumor suppressor protein, p53 [57]. However, there is currently no study focused on the significance of ACOX1 and LITAF in human CRC, so they are worthy of further study. Furthermore, GSEA analysis revealed that 
all of these key mRNAs were significantly enriched in well-known tumorigenesis related pathways, such as JAK-STAT signaling pathway [58], TGF- $\beta$ signaling pathway [59], ERBB signaling pathway [60], and VEGF signaling pathway [61]. These reports above partially improved the credibility of our research, and further research on the significance of the novel ceRNA network in CRC is valuable.

Tumor microenvironment (TME), a complex system which is composed of tumor cells, stromal cells, immune cells, fibroblasts, blood vessels, and cytokines, has been reported to regulate some well-known oncogenic process including apoptosis, angiogenesis, hypoxia, and immune escape [62]. Immune cells are important components of TME which induced the host immune response by secreting cytokines, thereby inhibiting or promoting the progression of tumor cells [63]. For example, the regulatory $T$ cells (Tregs) secrete immunosuppressive cytokines TGF- $\beta$ and interleukin-10 (IL-10) to protect cancer cells from cytotoxic $T$ cell response and induce immune tolerance, while $C D 8^{+} \mathrm{T}$ cells secrete interleukin-2 (IL2), tumor necrosis factor a (TNF-a) and interferon-y (IFN-y) to kill cancer cells in the TME [64]. Immunotherapy has shown encouraging results as an emerging therapy for some cancers. However, existing evidence suggested that the majority of CRC patients were insensitive to immunotherapy compared with esophageal cancer and lung cancer. Therefore, exploring the infiltration abundance and function of immune cells are important to enhance the efficacy of immunotherapy in CRC. In the present study, we found that the ceRNA regulatory network is significantly associated with the infiltration of immune cells, including $\mathrm{CD} 4^{+} / \mathrm{CD} 8^{+} \mathrm{T}$ cells and Macrophages. Importantly, we found that the ceRNA regulatory network is significantly correlated with the expression of some well-known immune checkpoints. Accumulating studies have reported that tumor infiltrating lymphocytes, including $\mathrm{CD} 4^{+}$and $\mathrm{CD}^{+} \mathrm{T}$ cells are important components of immune cells with anti-tumor functions. Furthermore, immune cells are highly heterogeneous in TME compared with noncancerous areas, infiltration of tumor infiltrating lymphocytes were associated with improved survival in some cases [63]. Studies also have indicated that ligands of immune checkpoints are highly expressed in cancer cells and TME, leading to T cells' functional exhaustion and T cell failure, thereby inducing immune escape of CRC cells in the TME [65]. Based on previous studies and our results above, we speculate that the survival related ceRNA regulatory network identified by our study may play an important role in immune escape of cancer cells in CRC TME, further study on this survival related ceRNA network will help to improve the efficacy of immunotherapy in CRC.

Some limitations are inevitably existed in the present study. First and foremost, all conclusions of our study were based online databases such as TCGA and GEO, further studies will be needed to validate our findings, and to elucidate whether and how the survival related ceRNA regulatory network regulates CRC. Second, we did not evaluate the subtype of CRC, such as mismatch repair (MMR) and microsatellite instability (MSI) status, which may affect the expression profiles and prognosis of CRC patients. Third, we can not obtain all the clinicopathologic data of each patient, and the survival analysis was performed by online database automatically, which may lead to the happen of bias.

\section{Conclusion}


In conclusion, we successfully constructed a survival related ceRNA regulatory network in CRC by means of stepwise prediction and validation, and each component of the ceRNA regulatory network was significantly correlated with the prognosis of patients with CRC. More importantly, immune infiltration analysis revealed that the survival related ceRNA regulatory network was remarkably associated with infiltration abundance of multiple immune cells and expression levels of immune checkpoints. Our findings provide valuable clues into improving the efficacy of targeted therapy and immunotherapy for human CRC.

\section{Abbreviations}

BP: Biological processes; CC: Cellular component; CCLE: Cancer Cell Line Encyclopedia; ceRNA:

Competing endogenous RNA; CRC: Colorectal cancer; DAVID: Database for Annotation, Visualization and Integrated Discovery; EMT: Epithelial mesenchymal transition; GEDS: Gene Expression Display Server; GEO: Gene Expression Omnibus; GEGPIA: Gene Expression Profiling Interactive Analysis; GETx: Genotype Tissue Expression; GO: Gene Ontology; GSEA: Gene Set Enrichment Analysis; IFN-ү: Interferon-ү; IL-2: Interleukin-2; IL-10: Interleukin-10; KEGG: Kyoto Encyclopedia of Genes and Genomes; LncRNA: Long noncoding RNA; MCLP: MD Anderson Cell Lines Project; MF: Molecular function; miRNA: microRNA; MMP: Matrix metalloproteinase; MMR: Mismatch repair; MSI: Microsatellite instability; TCGA: The Cancer Genome Atlas; TIMER: Tumor Immune Estimation Resource; TME: Tumor microenvironment; TNF-a: Tumor necrosis factor $\mathrm{a}$.

\section{Declarations}

\section{Ethics approval and consent to participate}

Not applicable.

\section{Consent for publication}

Not applicable.

\section{Availability of data and materials}

The datasets generated and/or analyzed during the current study are available in the Gene Expression Omnibus (GEO) repository (https://www.ncbi.nlm.nih.gov/geo/) and The Cancer Genome Atlas (TCGA, https://gdc-portal.nci.nih.gov/) databases.

\section{Competing interests}

The authors declare that they have no known competing financial interests or personal relationships that could have appeared to influence the work reported in this paper.

\section{Funding}


No funding was received.

\section{Authors' contributions}

$\mathrm{JL}$ and QY conceived the present study. $\mathrm{JL}, \mathrm{TH}, \mathrm{YW}$ and $\mathrm{XW}$ performed the bioinformatics analysis and interpretation of the data. JL and TH drafted the manuscript. QY agreed to be responsible for all aspects of the work to ensure that issues of accuracy or completeness of the study were properly investigated and addressed. All authors read and approved the final manuscript.

\section{Acknowledgements}

Not applicable.

\section{References}

1. Bray F, Ferlay J, Soerjomataram I, Siegel RL, Torre LA, Jemal A. Global cancer statistics 2018 : GLOBOCAN estimates of incidence and mortality worldwide for 36 cancers in 185 countries. CA Cancer J Clin. 2018;68(6):394-424. doi:10.3322/caac.21492

2. Siegel RL, Miller KD, Jemal A. Cancer statistics, 2020. CA Cancer J Clin. 2020;70(1):7-30. doi:10.3322/caac. 21590

3. Miller KD, Nogueira L, Mariotto AB, et al. Cancer treatment and survivorship statistics, 2019. CA Cancer J Clin. 2019;69(5):363-385. doi:10.3322/caac.21565

4. Van Cutsem E, Grothey A. Regorafenib for metastatic colorectal cancer - Authors' reply. Lancet. 2013;381(9877):1538-1539. doi:10.1016/S0140-6736(13)60978-2

5. Zhu Y, Bian Y, Zhang Q, et al. Construction and analysis of dysregulated IncRNA-associated ceRNA network in colorectal cancer. J Cell Biochem. 2019;120(6):9250-9263. doi:10.1002/jcb.28201

6. Donadon M, Ribero D, Morris-Stiff G, Abdalla EK, Vauthey JN. New paradigm in the management of liver-only metastases from colorectal cancer. Gastrointest Cancer Res. 2007;1(1):20-27.

7. Wang Y, Lu JH, Wu QN, et al. LncRNA LINRIS stabilizes IGF2BP2 and promotes the aerobic glycolysis in colorectal cancer. Mol Cancer. 2019;18(1):174. Published 2019 Dec 2. doi:10.1186/s12943-0191105-0

8. Bian Z, Zhang J, Li M, et al. LncRNA-FEZF1-AS1 Promotes Tumor Proliferation and Metastasis in Colorectal Cancer by Regulating PKM2 Signaling. Clin Cancer Res. 2018;24(19):4808-4819. doi:10.1158/1078-0432.CCR-17-2967

9. Wei GH, Wang X. IncRNA MEG3 inhibit proliferation and metastasis of gastric cancer via p53 signaling pathway. Eur Rev Med Pharmacol Sci. 2017;21(17):3850-3856.

10. Xue X, Yang YA, Zhang A, et al. LncRNA HOTAIR enhances ER signaling and confers tamoxifen resistance in breast cancer. Oncogene. 2016;35(21):2746-2755. doi:10.1038/onc.2015.340

11. Li X, Wu Z, Fu X, Han W. Long Noncoding RNAs: Insights from Biological Features and Functions to Diseases. Med Res Rev. 2013;33(3):517-553. doi:10.1002/med.21254 
12. Salmena L, Poliseno L, Tay Y, Kats L, Pandolfi PP. A ceRNA hypothesis: the Rosetta Stone of a hidden RNA language?. Cell. 2011;146(3):353-358. doi:10.1016/j.cell.2011.07.014

13. Fang $L$, Du WW, Yang $X$, et al. Versican 3'-untranslated region (3'-UTR) functions as a ceRNA in inducing the development of hepatocellular carcinoma by regulating miRNA activity. FASEB J. 2013;27(3):907-919. doi:10.1096/fj.12-220905

14. Lu JH, Zuo ZX, Wang W, et al. A two-microRNA-based signature predicts first-line chemotherapy outcomes in advanced colorectal cancer patients. Cell Death Discov. 2018;4:116. Published 2018 Dec 18. doi:10.1038/s41420-018-0133-7

15. Edgar R, Domrachev M, Lash AE. Gene Expression Omnibus: NCBI gene expression and hybridization array data repository. Nucleic Acids Res. 2002;30(1):207-210. doi:10.1093/nar/30.1.207

16. Ritchie ME, Phipson B, Wu D, et al. limma powers differential expression analyses for RNAsequencing and microarray studies. Nucleic Acids Res. 2015;43(7):e47. doi:10.1093/nar/gkv007

17. Xia M, Liu CJ, Zhang Q, Guo AY. GEDS: A Gene Expression Display Server for mRNAs, miRNAs and Proteins. Cells. 2019;8(7):675. Published 2019 Jul 3. doi:10.3390/cells8070675

18. Li JH, Liu S, Zhou H, Qu LH, Yang JH. starBase v2.0: decoding miRNA-ceRNA, miRNA-ncRNA and protein-RNA interaction networks from large-scale CLIP-Seq data. Nucleic Acids Res. 2014;42(Database issue):D92-D97. doi:10.1093/nar/gkt1248

19. Paraskevopoulou MD, Vlachos IS, Karagkouni D, et al. DIANA-LncBase v2: indexing microRNA targets on non-coding transcripts. Nucleic Acids Res. 2016;44(D1):D231-D238. doi:10.1093/nar/gkv1270

20. Chou $\mathrm{CH}$, Shrestha S, Yang CD, et al. miRTarBase update 2018: a resource for experimentally validated microRNA-target interactions. Nucleic Acids Res. 2018;46(D1):D296-D302. doi:10.1093/nar/gkx1067

21. Lewis BP, Shih IH, Jones-Rhoades MW, Bartel DP, Burge CB. Prediction of mammalian microRNA targets. Cell. 2003;115(7):787-798. doi:10.1016/s0092-8674(03)01018-3

22. Chen $Y$, Wang $X$. miRDB: an online database for prediction of functional microRNA targets. Nucleic Acids Res. 2020;48(D1):D127-D131. doi:10.1093/nar/gkz757

23. Shannon P, Markiel A, Ozier O, et al. Cytoscape: a software environment for integrated models of biomolecular interaction networks. Genome Res. 2003;13(11):2498-2504. doi:10.1101/gr.1239303

24. Huang da W, Sherman BT, Lempicki RA. Systematic and integrative analysis of large gene lists using DAVID bioinformatics resources. Nat Protoc. 2009;4(1):44-57. doi:10.1038/nprot.2008.211

25. Tang Z, Li C, Kang B, Gao G, Li C, Zhang Z. GEPIA: a web server for cancer and normal gene expression profiling and interactive analyses. Nucleic Acids Res. 2017;45(W1):W98-W102. doi:10.1093/nar/gkx247

26. Mizuno H, Kitada K, Nakai K, Sarai A. PrognoScan: a new database for meta-analysis of the prognostic value of genes. BMC Med Genomics. 2009;2:18. Published 2009 Apr 24. doi:10.1186/1755-8794-2-18 
27. Subramanian A, Tamayo P, Mootha VK, et al. Gene set enrichment analysis: a knowledge-based approach for interpreting genome-wide expression profiles. Proc Natl Acad Sci U S A. 2005;102(43):15545-15550. doi:10.1073/pnas.0506580102

28. Li T, Fan J, Wang B, et al. TIMER: A Web Server for Comprehensive Analysis of Tumor-Infiltrating Immune Cells. Cancer Res. 2017;77(21):e108-e110. doi:10.1158/0008-5472.CAN-17-0307

29. Han P, Li JW, Zhang BM, et al. The IncRNA CRNDE promotes colorectal cancer cell proliferation and chemoresistance via miR-181a-5p-mediated regulation of Wnt/ $\beta$-catenin signaling. Mol Cancer. 2017;16(1):9. Published 2017 Jan 13. doi:10.1186/s12943-017-0583-1

30. Xu M, Chen X, Lin K, et al. The long noncoding RNA SNHG1 regulates colorectal cancer cell growth through interactions with EZH2 and miR-154-5p. Mol Cancer. 2018;17(1):141. Published 2018 Sep 28. doi:10.1186/s12943-018-0894-x

31. Xu J, Meng Q, Li X, et al. Long Noncoding RNA MIR17HG Promotes Colorectal Cancer Progression via miR-17-5p. Cancer Res. 2019;79(19):4882-4895. doi:10.1158/0008-5472.CAN-18-3880

32. Luo J, Yao JF, Deng XF, et al. 14, 15-EET induces breast cancer cell EMT and cisplatin resistance by up-regulating integrin av $\beta 3$ and activating FAK/PI3K/AKT signaling. J Exp Clin Cancer Res. 2018;37(1):23. Published 2018 Feb 9. doi:10.1186/s13046-018-0694-6

33. Farhan M, Wang H, Gaur U, Little PJ, Xu J, Zheng W. FOXO Signaling Pathways as Therapeutic Targets in Cancer. Int J Biol Sci. 2017;13(7):815-827. Published 2017 Jul 6. doi:10.7150/ijbs.20052

34. Zhan T, Rindtorff N, Boutros M. Wnt signaling in cancer. Oncogene. 2017;36(11):1461-1473. doi:10.1038/onc.2016.304

35. Xu F, Cui WQ, Wei Y, et al. Astragaloside IV inhibits lung cancer progression and metastasis by modulating macrophage polarization through AMPK signaling. J Exp Clin Cancer Res. 2018;37(1):207. Published 2018 Aug 29. doi:10.1186/s13046-018-0878-0

36. Mattick JS, Rinn JL. Discovery and annotation of long noncoding RNAs. Nat Struct Mol Biol. 2015;22(1):5-7. doi:10.1038/nsmb.2942

37. Augoff K, McCue B, Plow EF, Sossey-Alaoui K. miR-31 and its host gene IncRNA LOC554202 are regulated by promoter hypermethylation in triple-negative breast cancer. Mol Cancer. 2012;11:5. Published 2012 Jan 30. doi:10.1186/1476-4598-11-5

38. Chen DL, Chen LZ, Lu YX, et al. Long noncoding RNA XIST expedites metastasis and modulates epithelial-mesenchymal transition in colorectal cancer. Cell Death Dis. 2017;8(8):e3011. Published 2017 Aug 24. doi:10.1038/cddis.2017.421

39. Thiery JP, Acloque H, Huang RY, Nieto MA. Epithelial-mesenchymal transitions in development and disease. Cell. 2009;139(5):871-890. doi:10.1016/j.cell.2009.11.007

40. Zhu J, Zhang R, Yang D, et al. Knockdown of Long Non-Coding RNA XIST Inhibited Doxorubicin Resistance in Colorectal Cancer by Upregulation of miR-124 and Downregulation of SGK1. Cell Physiol Biochem. 2018;51(1):113-128. doi:10.1159/000495168

41. Zhang $M$, Weng $W$, Zhang $Q$, et al. The IncRNA NEAT1 activates Wnt/ $\beta$-catenin signaling and promotes colorectal cancer progression via interacting with DDX5. J Hematol Oncol. 2018;11(1):113. 
Published 2018 Sep 5. doi:10.1186/s13045-018-0656-7

42. Wang X, Jiang G, Ren W, Wang B, Yang C, Li M. LncRNA NEAT1 Regulates 5-Fu Sensitivity, Apoptosis and Invasion in Colorectal Cancer Through the MiR-150-5p/CPSF4 Axis. Onco Targets Ther. 2020;13:6373-6383. Published 2020 Jul 1. doi:10.2147/OTT.S239432

43. Inui M, Martello G, Piccolo S. MicroRNA control of signal transduction. Nat Rev Mol Cell Biol. 2010;11(4):252-263. doi:10.1038/nrm2868

44. Mansoori B, Mohammadi A, Naghizadeh S, et al. miR-330 suppresses EMT and induces apoptosis by downregulating HMGA2 in human colorectal cancer. J Cell Physiol. 2020;235(2):920-931. doi:10.1002/jcp.29007

45. Liu T, Zhang X, Du L, et al. Exosome-transmitted miR-128-3p increase chemosensitivity of oxaliplatinresistant colorectal cancer [published correction appears in Mol Cancer. 2020 May 12;19(1):89]. Mol Cancer. 2019;18(1):43. Published 2019 Mar 19. doi:10.1186/s12943-019-0981-7

46. Sun M, Song H, Wang S, et al. Integrated analysis identifies microRNA-195 as a suppressor of HippoYAP pathway in colorectal cancer. J Hematol Oncol. 2017;10(1):79. Published 2017 Mar 29. doi:10.1186/s13045-017-0445-8

47. Jin $Y$, Wang $M, H u$ H, Huang Q, Chen Y, Wang G. Overcoming stemness and chemoresistance in colorectal cancer through miR-195-5p-modulated inhibition of notch signaling. Int J Biol Macromol. 2018;117:445-453. doi:10.1016/j.ijbiomac.2018.05.151

48. Cheng $\mathrm{H}$, Malhotra A. Evaluation of Potential of Long Noncoding RNA NEAT1 in Colorectal Cancer. J Environ Pathol Toxicol Oncol. 2020;39(2):101-111.

doi:10.1615/JEnvironPatholToxicolOncol.2020032508

49. Yang C, Wu K, Wang S, Wei G. Long non-coding RNA XIST promotes osteosarcoma progression by targeting YAP via miR-195-5p. J Cell Biochem. 2018;119(7):5646-5656. doi:10.1002/jcb.26743

50. Zhang Q, Zhang C, Ma JX, Ren H, Sun Y, Xu JZ. Circular RNA PIP5K1A promotes colon cancer development through inhibiting miR-1273a. World J Gastroenterol. 2019;25(35):5300-5309. doi:10.3748/wjg.v25.i35.5300

51. Wu SH, Xu XY, Sun CB, et al. Expression of PHLPP2 correlates with clinicopathologic characteristics and prognosis in colorectal cancer. Int J Clin Exp Pathol. 2019;12(8):2909-2919. Published 2019 Aug 1.

52. Cui J, Cai Y, Hu Y, et al. Epigenetic silencing of TPM2 contributes to colorectal cancer progression upon RhoA activation. Tumour Biol. 2016;37(9):12477-12483. doi:10.1007/s13277-016-5103-1

53. Wei LJ, Bai DM, Wang ZY, Liu BC. MicroRNA-375 accelerates the invasion and migration of colorectal cancer through targeting RECK. Eur Rev Med Pharmacol Sci. 2019;23(11):4738-4745. doi:10.26355/eurrev_201906_18055

54. Shibao K, Fiedler MJ, Nagata J, et al. The type III inositol 1,4,5-trisphosphate receptor is associated with aggressiveness of colorectal carcinoma. Cell Calcium. 2010;48(6):315-323. doi:10.1016/j.ceca.2010.09.005 
55. Brown GT, Cash BG, Blihoghe D, Johansson P, Alnabulsi A, Murray GI. The expression and prognostic significance of retinoic acid metabolising enzymes in colorectal cancer. PLoS One. 2014;9(3):e90776. Published 2014 Mar 7. doi:10.1371/journal.pone.0090776

56. Zheng FM, Chen WB, Qin T, et al. ACOX1 destabilizes p73 to suppress intrinsic apoptosis pathway and regulates sensitivity to doxorubicin in lymphoma cells. BMB Rep. 2019;52(9):566-571. doi:10.5483/BMBRep.2019.52.9.094

57. Zou J, Guo P, Lv N, Huang D. Lipopolysaccharide-induced tumor necrosis factor-a factor enhances inflammation and is associated with cancer (Review). Mol Med Rep. 2015;12(5):6399-6404. doi:10.3892/mmr.2015.4243

58. Jiang L, Zhao XH, Mao YL, Wang JF, Zheng HJ, You QS. Long non-coding RNA RP11-468E2.5 curtails colorectal cancer cell proliferation and stimulates apoptosis via the JAK/STAT signaling pathway by targeting STAT5 and STAT6. J Exp Clin Cancer Res. 2019;38(1):465. Published 2019 Nov 12. doi:10.1186/s13046-019-1428-0

59. Li Y, Cao H, Jiao Z, et al. Carcinoembryonic antigen interacts with TGF-\{beta\} receptor and inhibits TGF-\{beta\} signaling in colorectal cancers. Cancer Res. 2010;70(20):8159-8168. doi:10.1158/00085472.CAN-10-1073

60. Dong W, Cao Z, Pang Y, Feng T, Tian H. CARF, As An Oncogene, Promotes Colorectal Cancer Stemness By Activating ERBB Signaling Pathway. Onco Targets Ther. 2019;12:9041-9051. Published 2019 Nov 1. doi:10.2147/OTT.S225733

61. Sun W, Wang X, Li J, et al. MicroRNA-181a promotes angiogenesis in colorectal cancer by targeting SRCIN1 to promote the SRC/VEGF signaling pathway. Cell Death Dis. 2018;9(4):438. Published 2018 Apr 1. doi:10.1038/s41419-018-0490-4

62. Casey SC, Amedei A, Aquilano K, et al. Cancer prevention and therapy through the modulation of the tumor microenvironment. Semin Cancer Biol. 2015;35 Suppl(Suppl):S199-S223. doi:10.1016/j.semcancer.2015.02.007

63. Ge P, Wang W, Li L, et al. Profiles of immune cell infiltration and immune-related genes in the tumor microenvironment of colorectal cancer. Biomed Pharmacother. 2019;118:109228. doi:10.1016/j.biopha.2019.109228

64. Xiong $Y$, Wang $Y$, Tiruthani $K$. Tumor immune microenvironment and nano-immunotherapeutics in colorectal cancer. Nanomedicine. 2019;21:102034. doi:10.1016/j.nano.2019.102034

65. Emambux S, Tachon G, Junca A, Tougeron D. Results and challenges of immune checkpoint inhibitors in colorectal cancer. Expert Opin Biol Ther. 2018;18(5):561-573.

doi:10.1080/14712598.2018.1445222

\section{Tables}


Table 1

Gene Ontology analysis of the ceRNA regulatory network

\begin{tabular}{|c|c|c|c|}
\hline Category & Term & Count & PValue \\
\hline GOTERM_BP_DIRECT & $\begin{array}{l}\text { GO:0045944 positive regulation of transcription } \\
\text { from RNA polymerase Il promoter }\end{array}$ & 40 & $1.07 \mathrm{E}-06$ \\
\hline GOTERM_BP_DIRECT & G0:0006468 protein phosphorylation & 24 & 4.97E-06 \\
\hline GOTERM_BP_DIRECT & G0:0016055 Wnt signaling pathway & 14 & $2.35 \mathrm{E}-05$ \\
\hline GOTERM_BP_DIRECT & $\begin{array}{l}\text { GO:0000122 negative regulation of transcription } \\
\text { from RNA polymerase II promoter }\end{array}$ & 28 & $1.43 \mathrm{E}-04$ \\
\hline GOTERM_BP_DIRECT & G0:0030336 negative regulation of cell migration & 9 & $2.44 \mathrm{E}-04$ \\
\hline GOTERM_BP_DIRECT & GO:0035264 multicellular organism growth & 8 & $4.71 \mathrm{E}-04$ \\
\hline GOTERM_BP_DIRECT & GO:0001657 ureteric bud development & 6 & 4.74E-04 \\
\hline GOTERM_BP_DIRECT & $\begin{array}{l}\text { G0:0030512 negative regulation of transforming } \\
\text { growth factor beta receptor signaling pathway }\end{array}$ & 7 & 8.30E-04 \\
\hline GOTERM_BP_DIRECT & GO:0045785 positive regulation of cell adhesion & 6 & $8.48 \mathrm{E}-04$ \\
\hline GOTERM_BP_DIRECT & $\begin{array}{l}\text { G0:0043066 negative regulation of apoptotic } \\
\text { process }\end{array}$ & 19 & 0.001023 \\
\hline GOTERM_CC_DIRECT & GO:0005654 nucleoplasm & 91 & $2.72 \mathrm{E}-11$ \\
\hline GOTERM_CC_DIRECT & GO:0005737 cytoplasm & 134 & $1.46 \mathrm{E}-09$ \\
\hline GOTERM_CC_DIRECT & Go:0005829 cytosol & 91 & $1.90 \mathrm{E}-07$ \\
\hline GOTERM_CC_DIRECT & GO:0005634 nucleus & 128 & $8.32 \mathrm{E}-07$ \\
\hline GOTERM_CC_DIRECT & GO:0016604 nuclear body & 5 & 0.001925 \\
\hline GOTERM_CC_DIRECT & G0:0035068 micro-ribonucleoprotein complex & 3 & 0.003777 \\
\hline GOTERM_CC_DIRECT & GO:0005770 late endosome & 8 & 0.0038 \\
\hline GOTERM_CC_DIRECT & G0:0070578 RISC-loading complex & 3 & 0.005232 \\
\hline GOTERM_CC_DIRECT & GO:0005913 cell-cell adherens junction & 13 & 0.006775 \\
\hline GOTERM_CC_DIRECT & G0:0000421 autophagosome membrane & 4 & 0.007488 \\
\hline GOTERM_MF_DIRECT & GO:0005515 protein binding & 215 & $2.47 \mathrm{E}-15$ \\
\hline GOTERM_MF_DIRECT & GO:0008013 beta-catenin binding & 11 & $1.25 \mathrm{E}-06$ \\
\hline GOTERM_MF_DIRECT & G0:0061630 ubiquitin protein ligase activity & 14 & 1.93E-05 \\
\hline GOTERM_MF_DIRECT & GO:0048185 activin binding & 5 & $3.64 \mathrm{E}-05$ \\
\hline GOTERM_MF_DIRECT & GO:0003682 chromatin binding & 20 & 4.27E-05 \\
\hline
\end{tabular}




\begin{tabular}{llll} 
GOTERM_MF_DIRECT & GO:0004672 $~$ protein kinase activity & 17 & $4.50 \mathrm{E}-04$ \\
GOTERM_MF_DIRECT & G0:0030165 PDZ domain binding & 8 & $6.52 \mathrm{E}-04$ \\
GOTERM_MF_DIRECT & GO:0004674 protein serine/threonine kinase activity & 17 & $7.43 \mathrm{E}-04$ \\
GOTERM_MF_DIRECT & GO:0019901 protein kinase binding & 17 & $7.43 \mathrm{E}-04$ \\
GOTERM_MF_DIRECT & GO:0043565 sequence-specific DNA binding & 20 & 0.001444 \\
\hline
\end{tabular}

Table 2

Kyoto Encyclopedia of Genes and Genomes pathway analysis of the ceRNA regulatory network

\begin{tabular}{|llll|}
\hline Category & Term & Count & PValue \\
\hline KEGG_PATHWAY & hsa05221:Acute myeloid leukemia & 8 & $9.08 \mathrm{E}-05$ \\
\hline KEGG_PATHWAY & hsa05212:Pancreatic cancer & 8 & $2.36 \mathrm{E}-04$ \\
\hline KEGG_PATHWAY & hsa05211:Renal cell carcinoma & 8 & $2.60 \mathrm{E}-04$ \\
\hline KEGG_PATHWAY & hsa05205:Proteoglycans in cancer & 13 & $4.60 \mathrm{E}-04$ \\
\hline KEGG_PATHWAY & hsa05200:Pathways in cancer & 19 & $5.18 \mathrm{E}-04$ \\
\hline KEGG_PATHWAY & hsa04151:PI3K-Akt signaling pathway & 17 & $9.45 \mathrm{E}-04$ \\
\hline KEGG_PATHWAY & hsa05222:Small cell lung cancer & 8 & 0.0012152 \\
\hline KEGG_PATHWAY & hsa04114:Oocyte meiosis & 9 & 0.0013139 \\
\hline KEGG_PATHWAY & hsa04910:Insulin signaling pathway & 10 & 0.001344 \\
\hline KEGG_PATHWAY & hsa04914:Progesterone-mediated oocyte maturation & 8 & 0.0013934 \\
\hline KEGG_PATHWAY & hsa04110:Cell cycle & 9 & 0.0026574 \\
\hline KEGG_PATHWAY & hsa04068:FoxO signaling pathway & 9 & 0.0042832 \\
\hline KEGG_PATHWAY & hsa04550:Signaling pathways regulating pluripotency of & 9 & 0.0055743 \\
\hline KEGG_PATHWAY & hsa05210:Colorectal cancer & 6 & 0.0067139 \\
\hline KEGG_PATHWAY & hsa04722:Neurotrophin signaling pathway & 8 & 0.0083948 \\
\hline KEGG_PATHWAY & hsa04152:AMPK signaling pathway & 8 & 0.0095582 \\
\hline KEGG_PATHWAY & hsa05220:Chronic myeloid leukemia & 6 & 0.012461 \\
\hline KEGG_PATHWAY & hsa05203:Viral carcinogenesis & 70 & 0.0175239 \\
\hline KEGG_PATHWAY & hsa05213:Endometrial cancer & 7 & 0.0176717 \\
\hline KEGG_PATHWAY & hsa04931:Insulin resistance & 6179023 \\
\hline
\end{tabular}


A

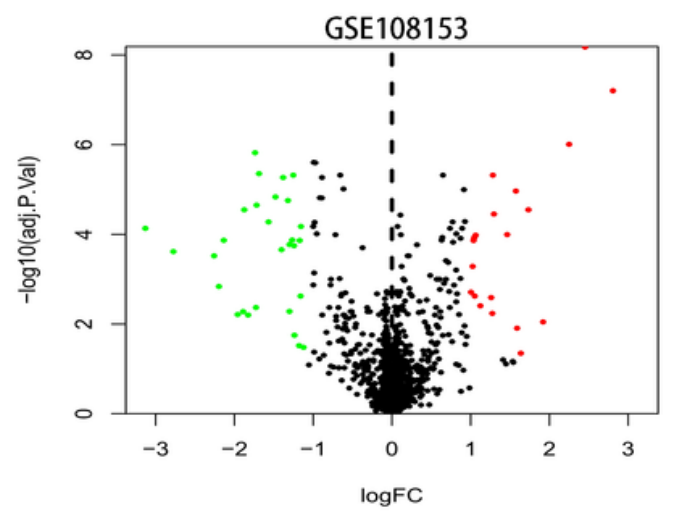

C

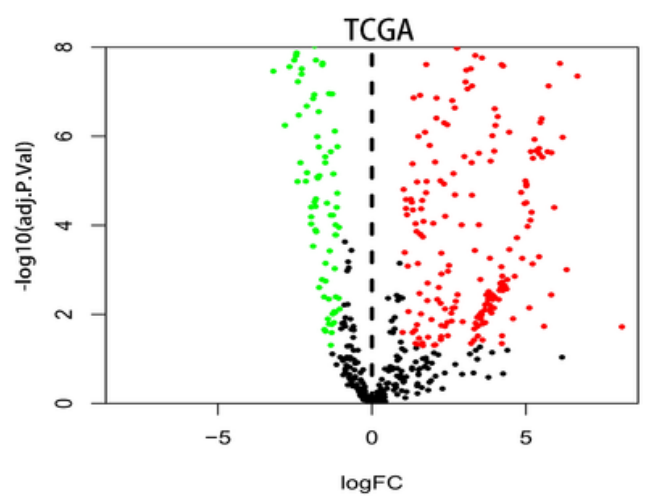

B

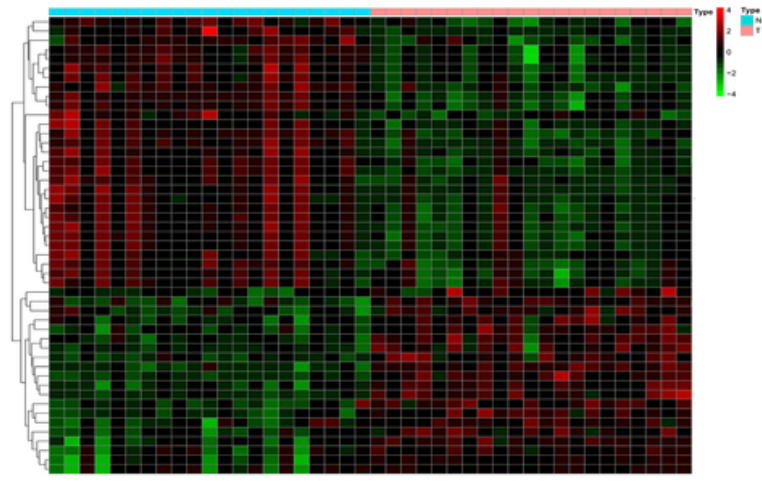

D

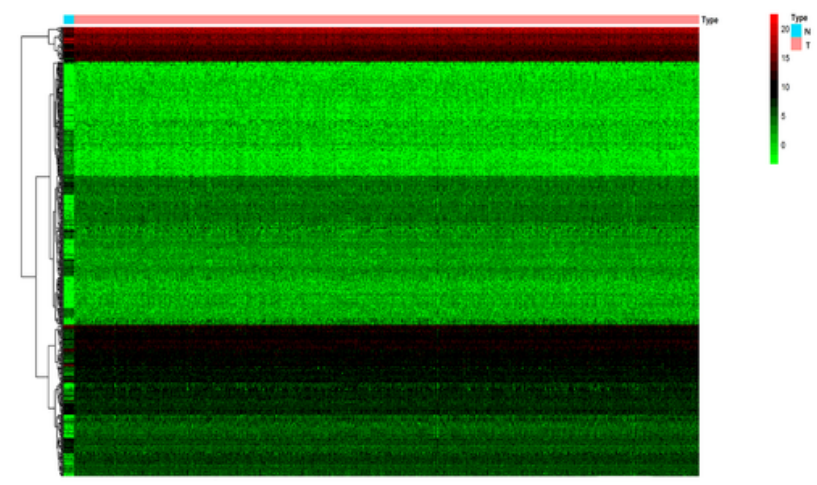

E TCGA-diff

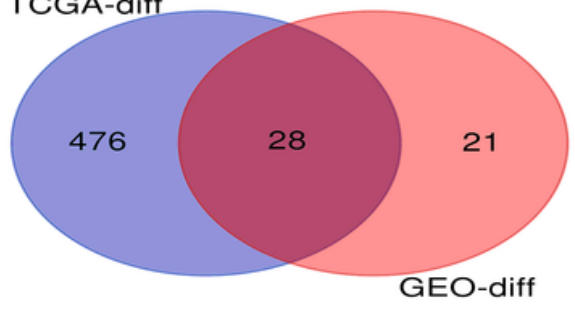

\section{Figure 1}

Identification of differentially expressed miRNA in human CRC. (A\&C) The volcano of differentially expressed miRNA in GSE108153 and TCGA datasets. The green dots and red dots represent the significantly downregulated and upregulated miRNAs, respectively. The black dots represent miRNAs with no significant difference. (B\&D) The heat map of differentially expressed miRNA in GSE108153 and TCGA datasets. (E) The intersection of differentially expressed miRNAs in GSE108153 and TCGA datasets, respectively. 
A

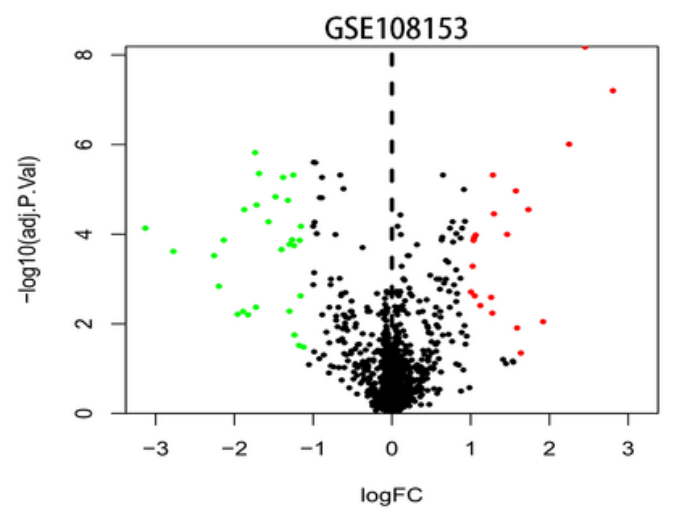

C

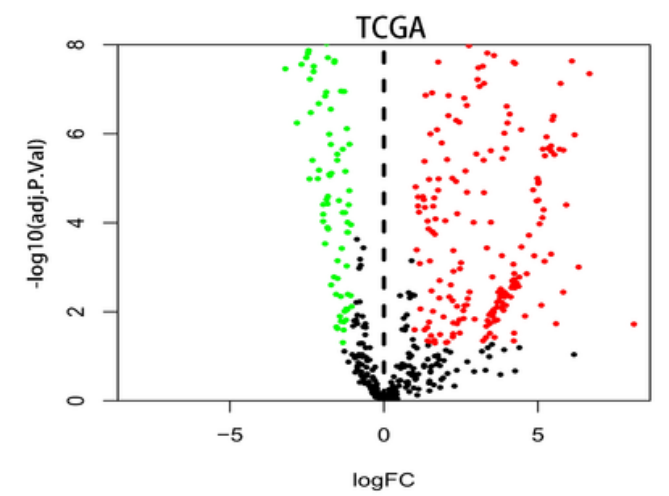

B

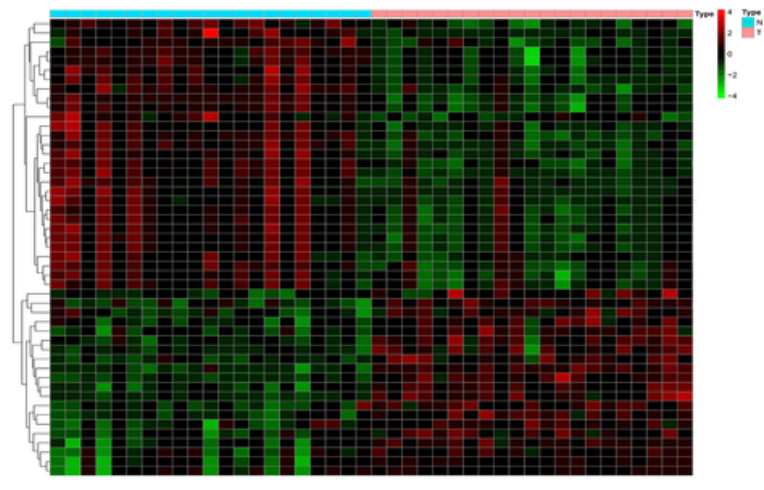

D

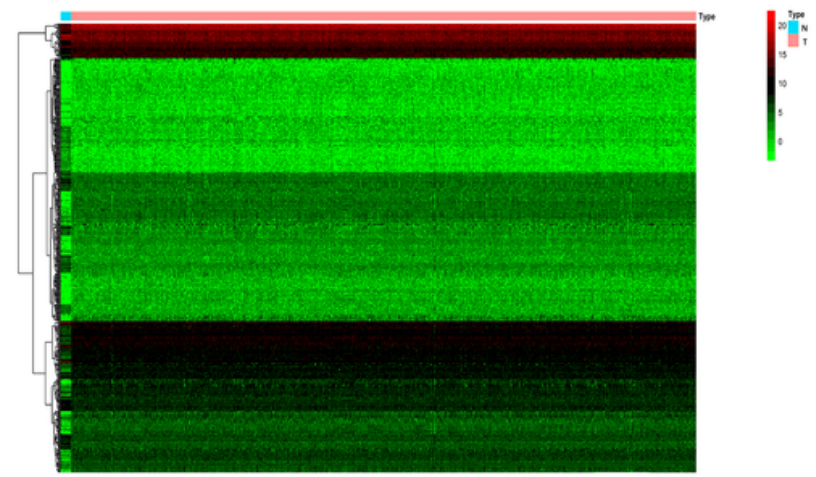

E TCGA-diff

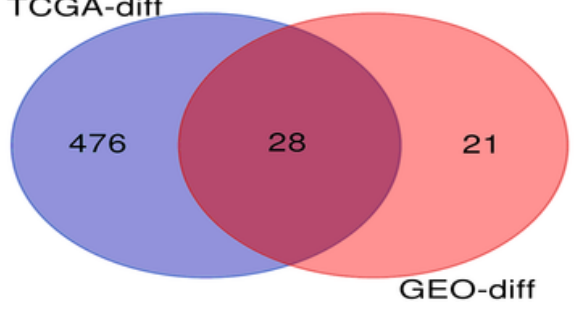

\section{Figure 1}

Identification of differentially expressed miRNA in human CRC. (A\&C) The volcano of differentially expressed miRNA in GSE108153 and TCGA datasets. The green dots and red dots represent the significantly downregulated and upregulated miRNAs, respectively. The black dots represent miRNAs with no significant difference. (B\&D) The heat map of differentially expressed miRNA in GSE108153 and TCGA datasets. (E) The intersection of differentially expressed miRNAs in GSE108153 and TCGA datasets, respectively. 

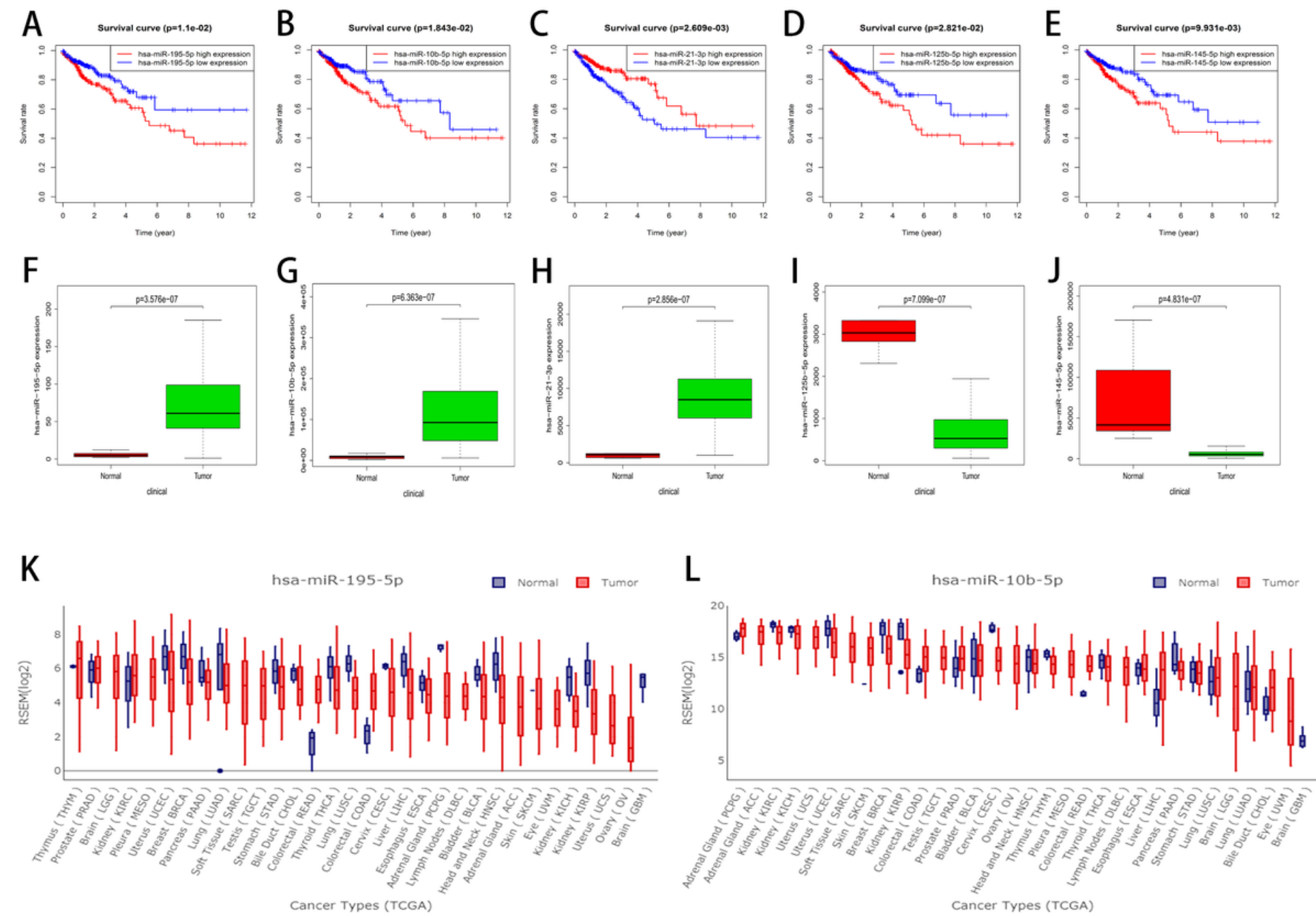

Figure 2

Identification of key miRNAs in CRC. (A-E) The Kaplan-Meier curve of the key miRNAs. (F-J) The expression box plot of the key miRNAs. (K\&L) The expression box plot of hsa-miR-9-5p and hsa-miR-10b$5 p$ in multiple types of cancer 

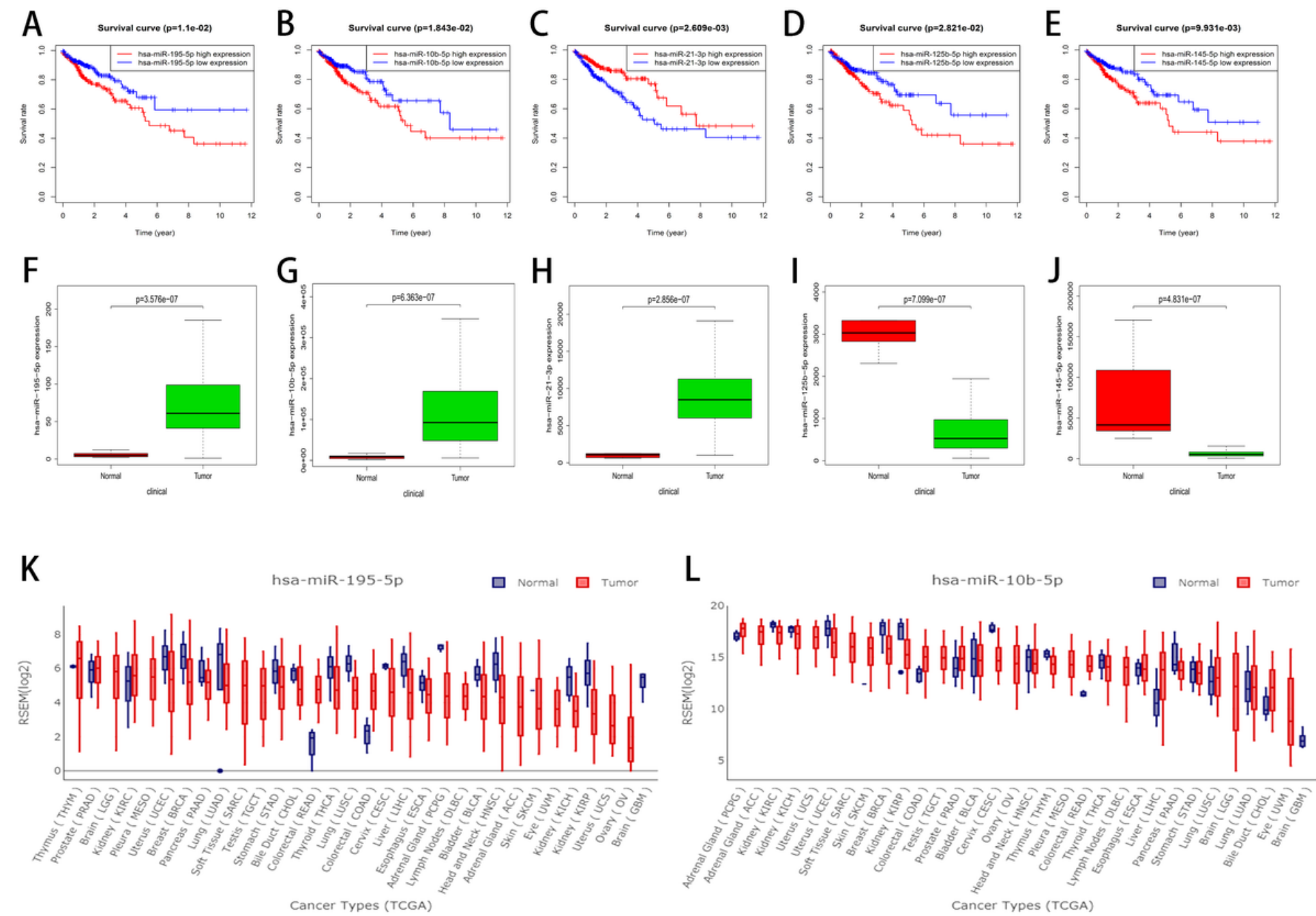

Figure 2

Identification of key miRNAs in CRC. (A-E) The Kaplan-Meier curve of the key miRNAs. (F-J) The expression box plot of the key miRNAs. (K\&L) The expression box plot of hsa-miR-9-5p and hsa-miR-10b$5 p$ in multiple types of cancer 
A

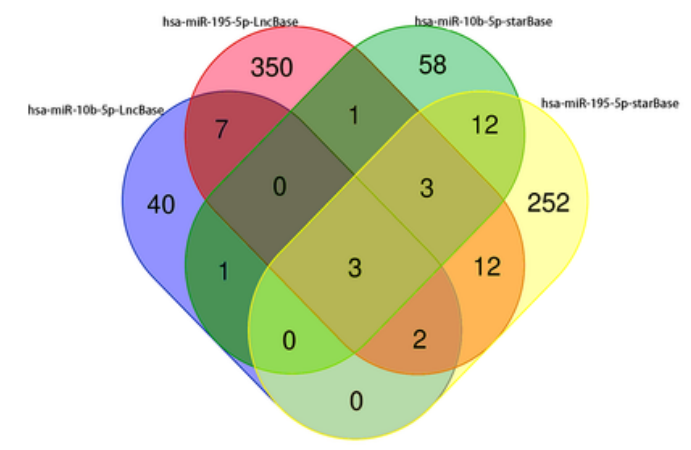

B

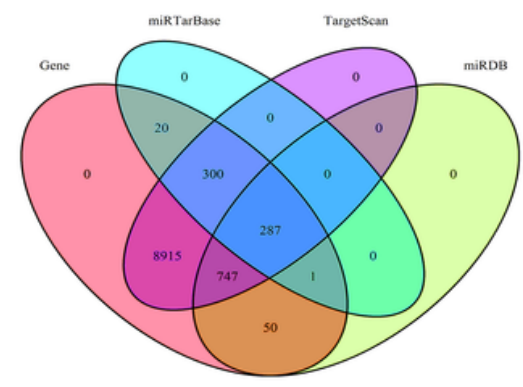

C

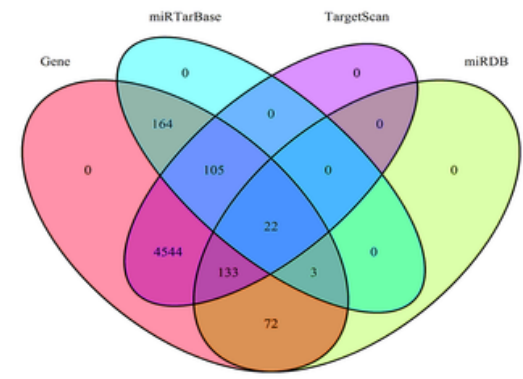

D

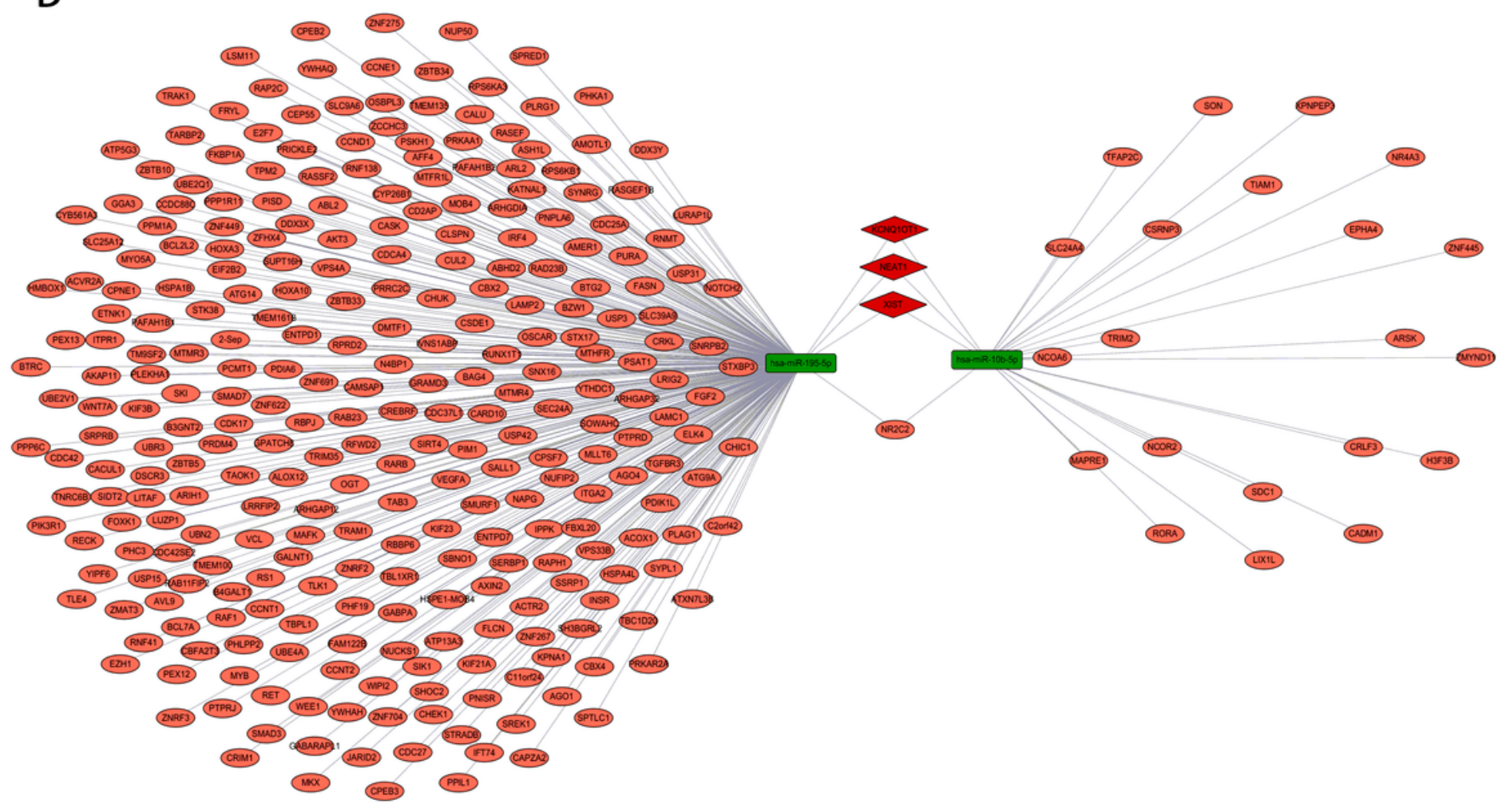

Figure 3

Identification of IncRNA-miRNA-mRNA regulatory network in CRC. (A) The intersection of upstream IncRNAs targeted by hsa-miR-9-5p and hsa-miR-10b-5p in LncBase v2 and starBase databases. (B\&C) The intersection of downstream mRNAs targeted by hsa-miR-9-5p and hsa-miR-10b-5p in miRTarBase, Targetscan, and miRDB databases. (D) The IncRNA-miRNA-mRNA regulatory network constructed by cytoscape software. The green ound rectangle in the network represents miRNA. The pink ellipse in the network represents mRNA. The red diamond in the network represents IncRNA. 
A

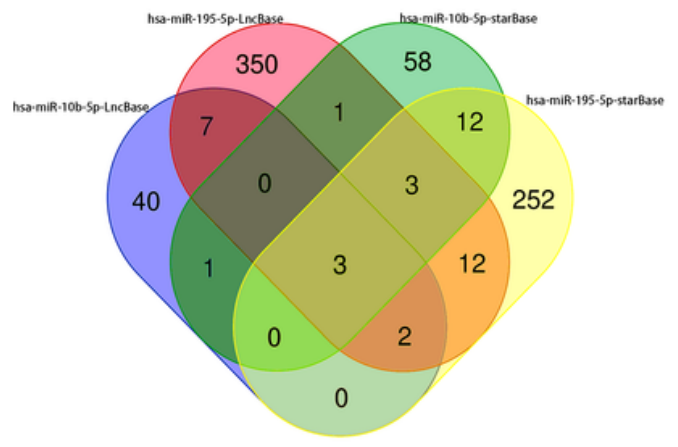

B

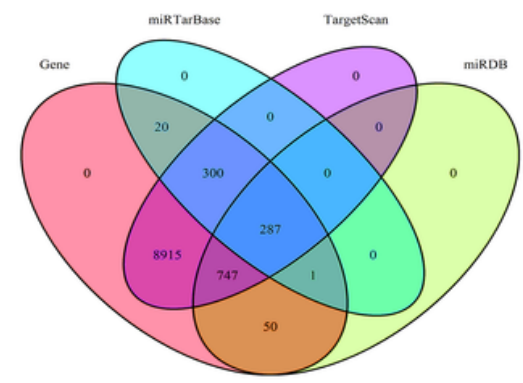

C

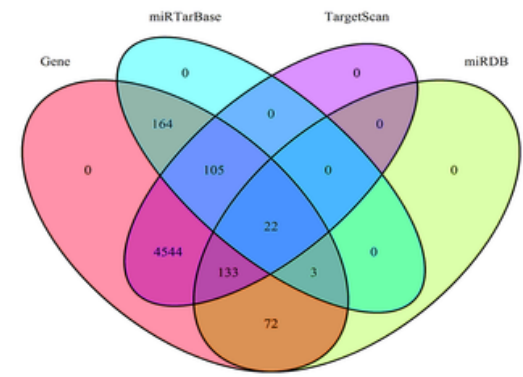

D

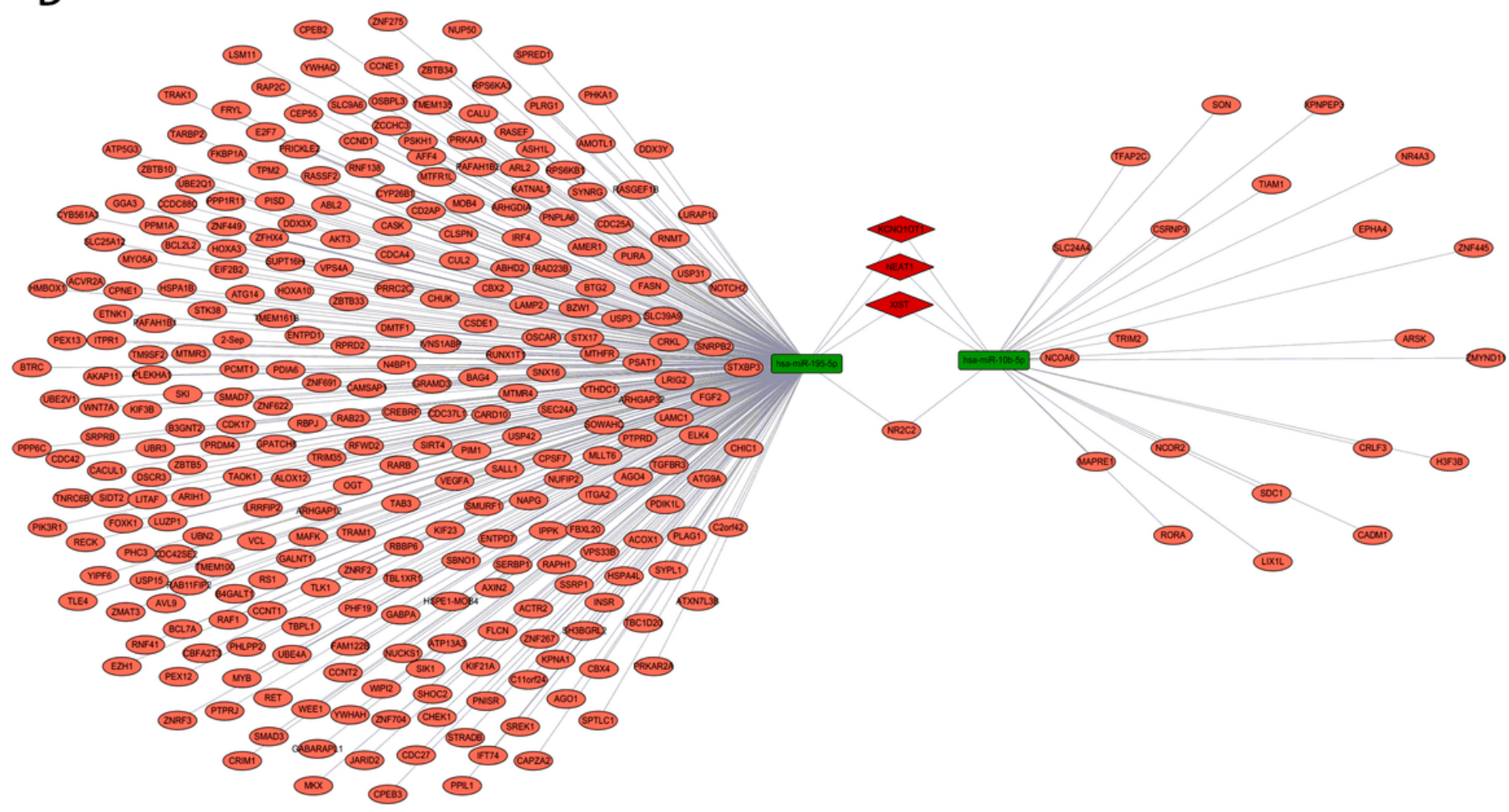

Figure 3

Identification of IncRNA-miRNA-mRNA regulatory network in CRC. (A) The intersection of upstream IncRNAs targeted by hsa-miR-9-5p and hsa-miR-10b-5p in LncBase v2 and starBase databases. (B\&C) The intersection of downstream mRNAs targeted by hsa-miR-9-5p and hsa-miR-10b-5p in miRTarBase, Targetscan, and miRDB databases. (D) The IncRNA-miRNA-mRNA regulatory network constructed by cytoscape software. The green ound rectangle in the network represents miRNA. The pink ellipse in the network represents mRNA. The red diamond in the network represents IncRNA. 
A
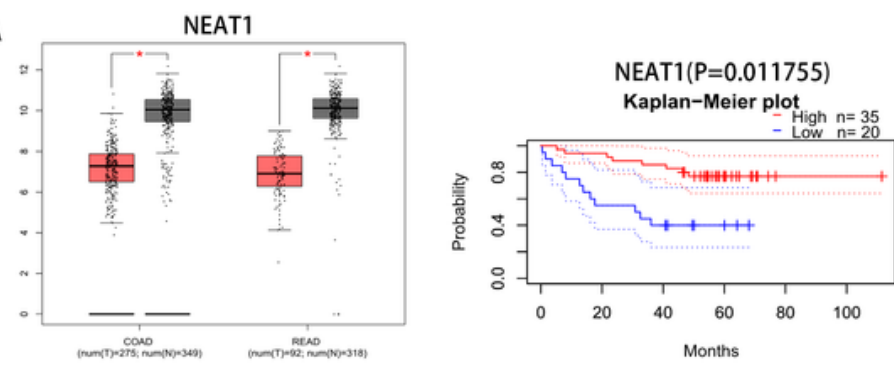

Croanten

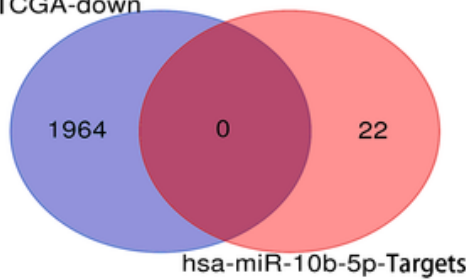

E

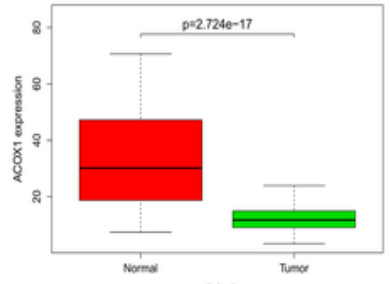

G

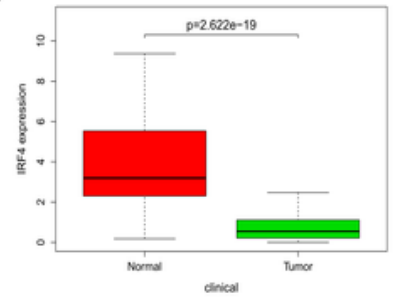

I

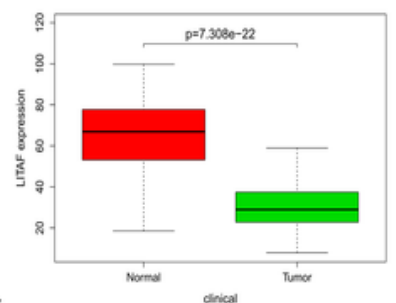

K

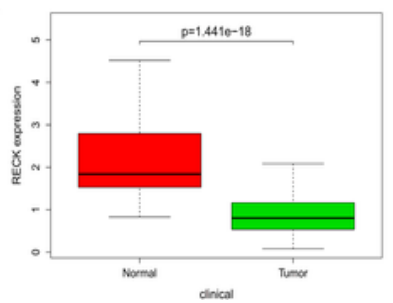

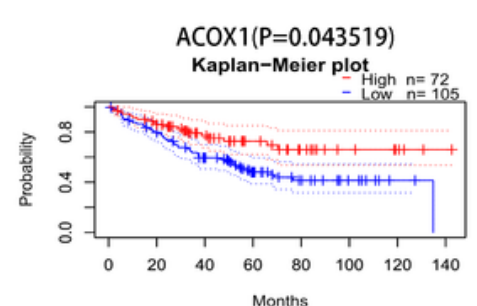
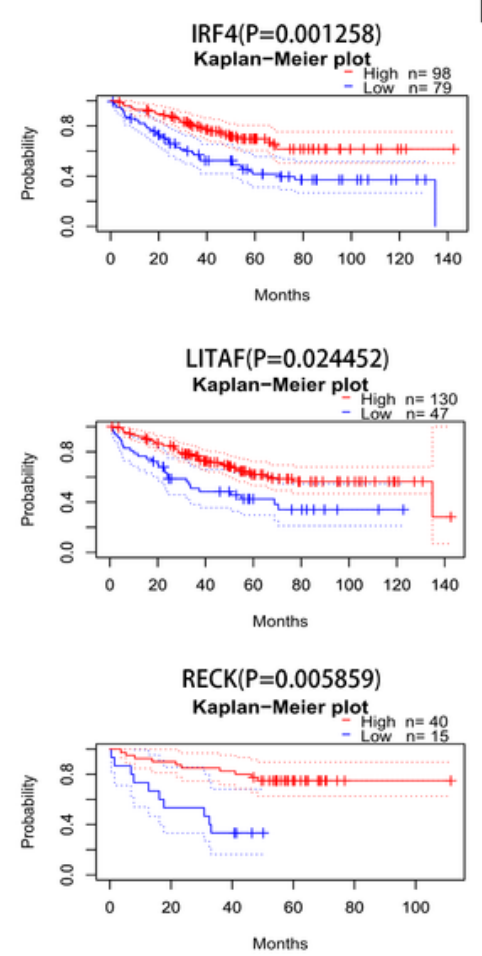

B
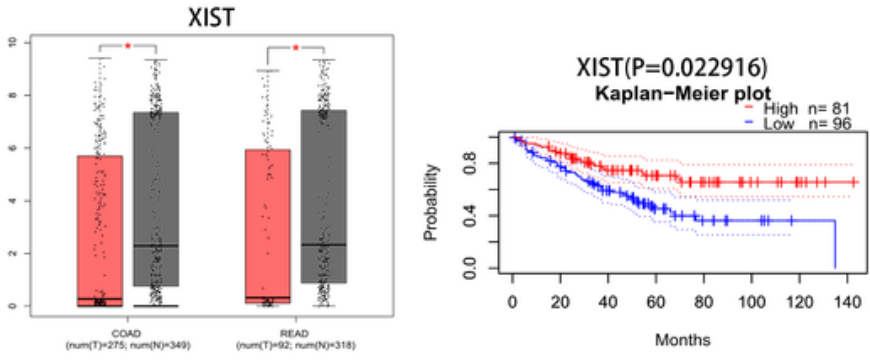

D TCGA-down

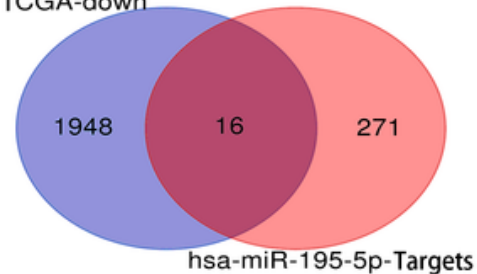

F
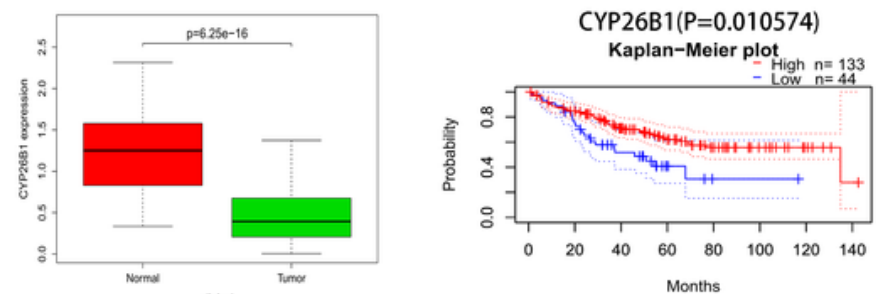

$\mathrm{H}$
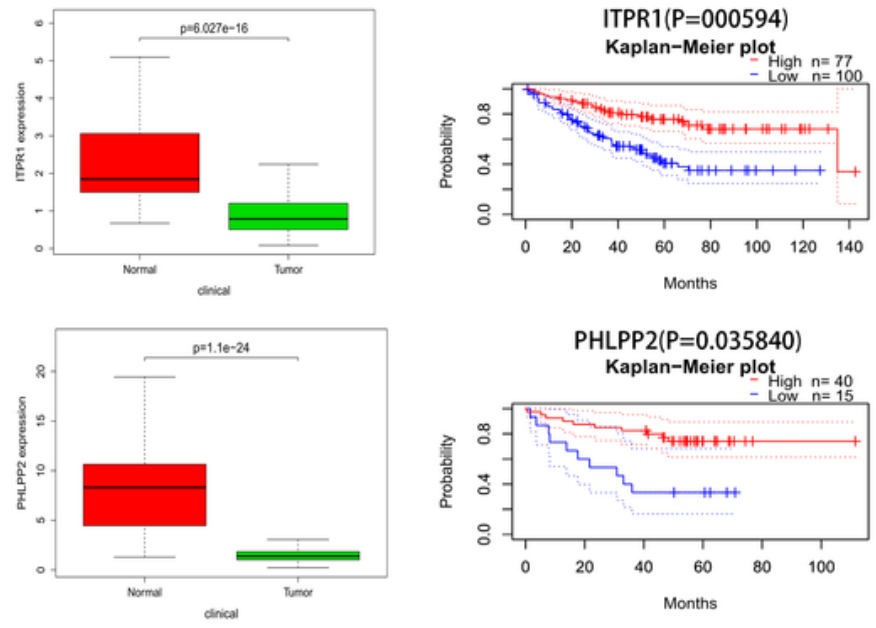

$\mathrm{L}$
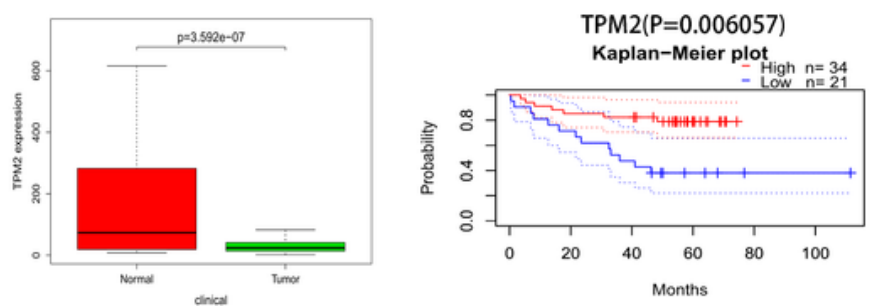

\section{Figure 4}

Identification of survival related IncRNA-miRNA-mRNA regulatory network in CRC. (A\&B) The expression levels and prognostic values of upstream IncRNA NEAT1 and XIST. (C\&D) The intersection of downstream mRNAs and down regulated mRNAs in TCGA datasets, respectively. (E) The expression levels and prognostic values of downstream mRNAs. 
A
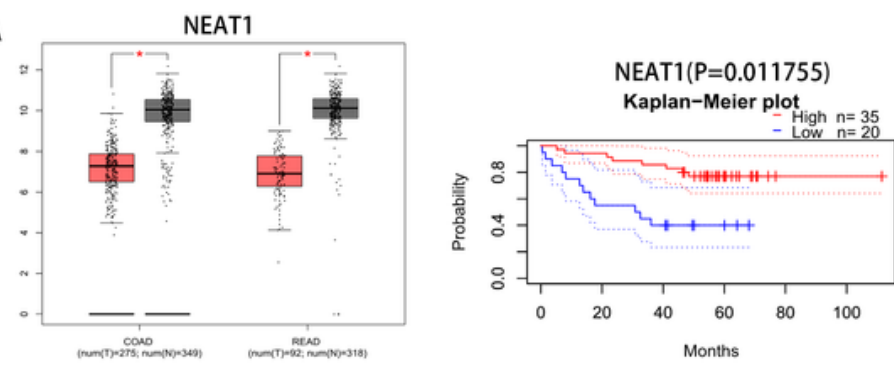

Croanten

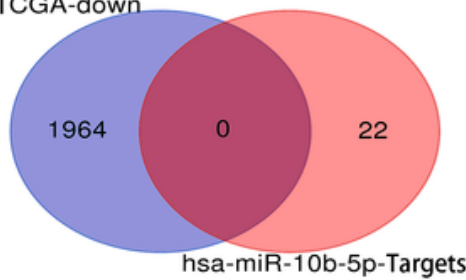

E

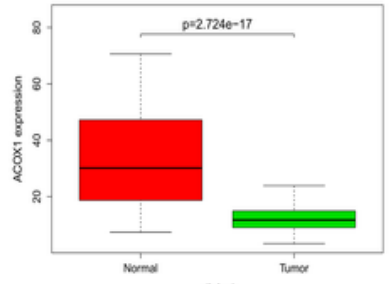

G

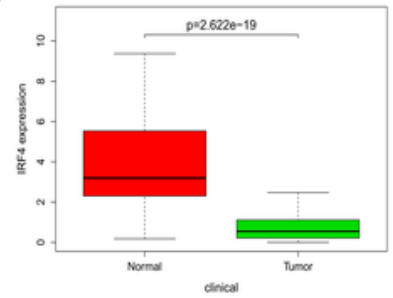

I

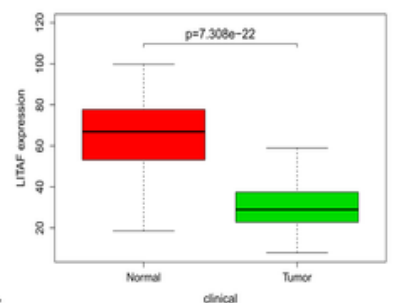

K

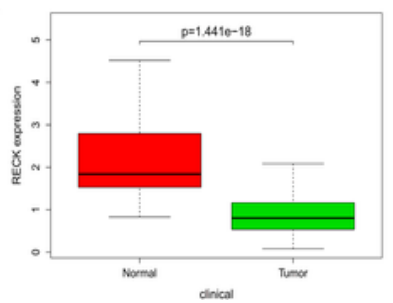

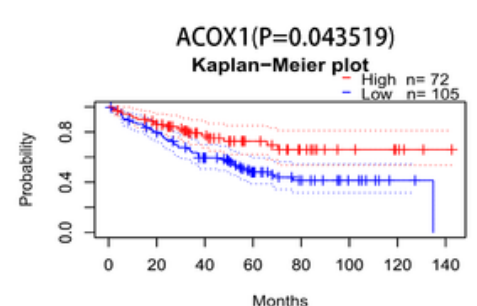
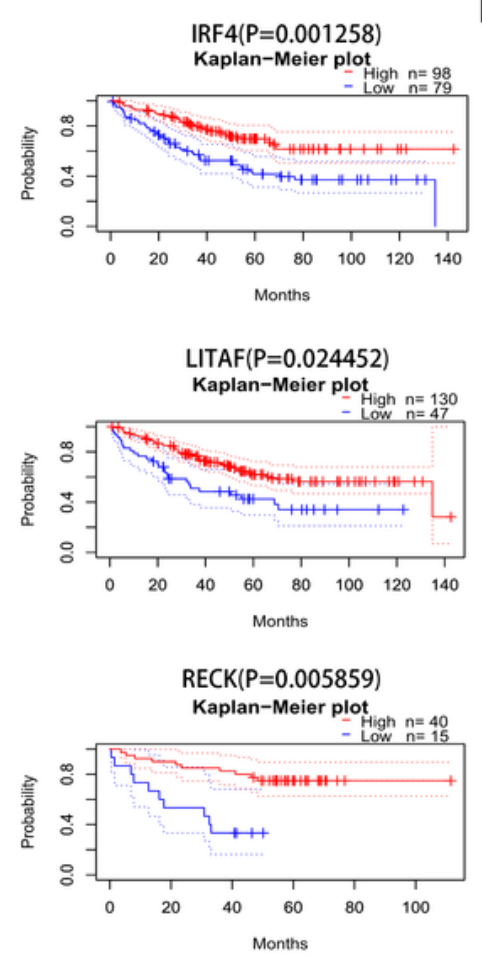

B
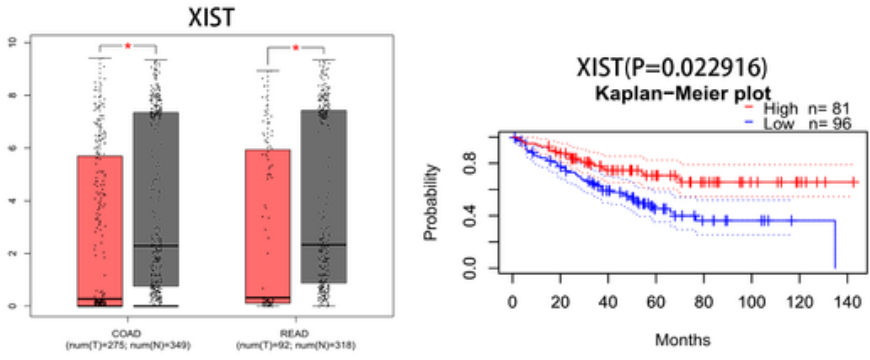

D TCGA-down

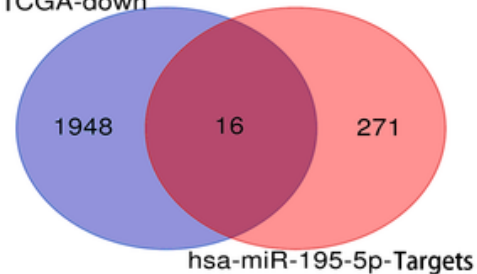

F
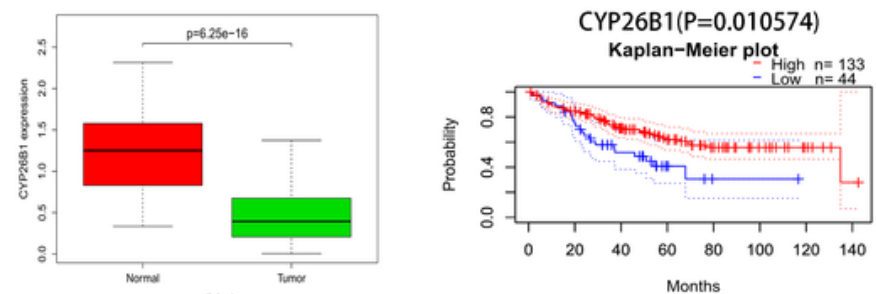

$\mathrm{H}$
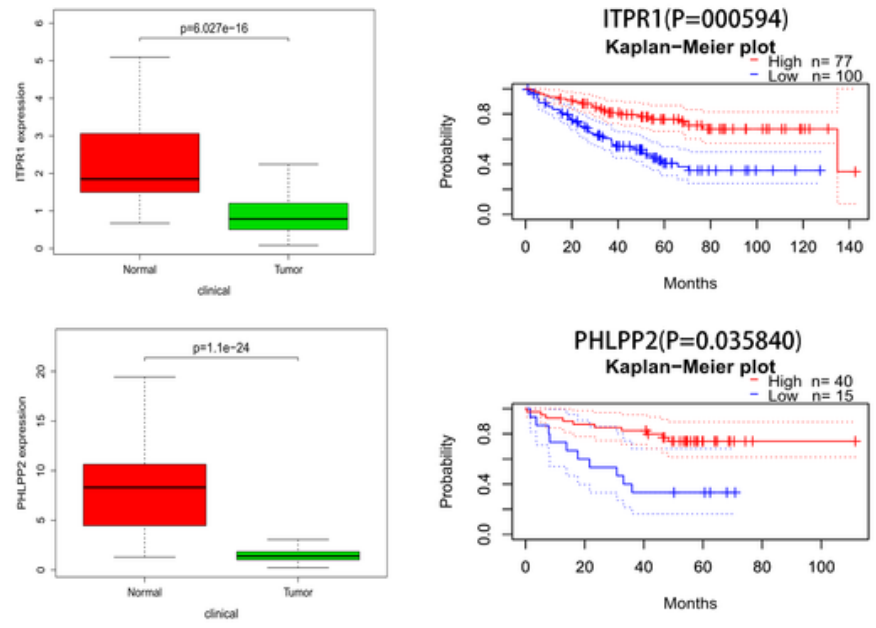

$\mathrm{L}$
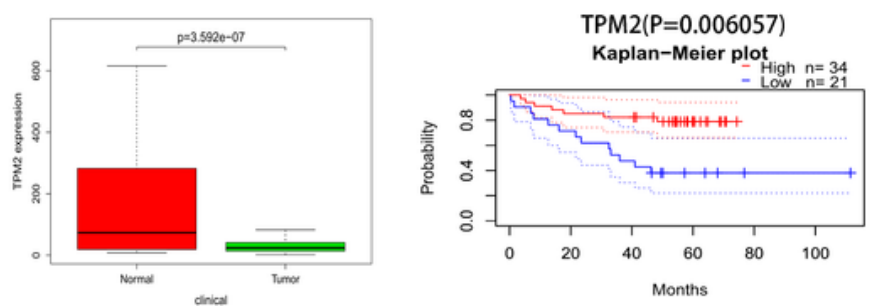

\section{Figure 4}

Identification of survival related IncRNA-miRNA-mRNA regulatory network in CRC. (A\&B) The expression levels and prognostic values of upstream IncRNA NEAT1 and XIST. (C\&D) The intersection of downstream mRNAs and down regulated mRNAs in TCGA datasets, respectively. (E) The expression levels and prognostic values of downstream mRNAs. 
A

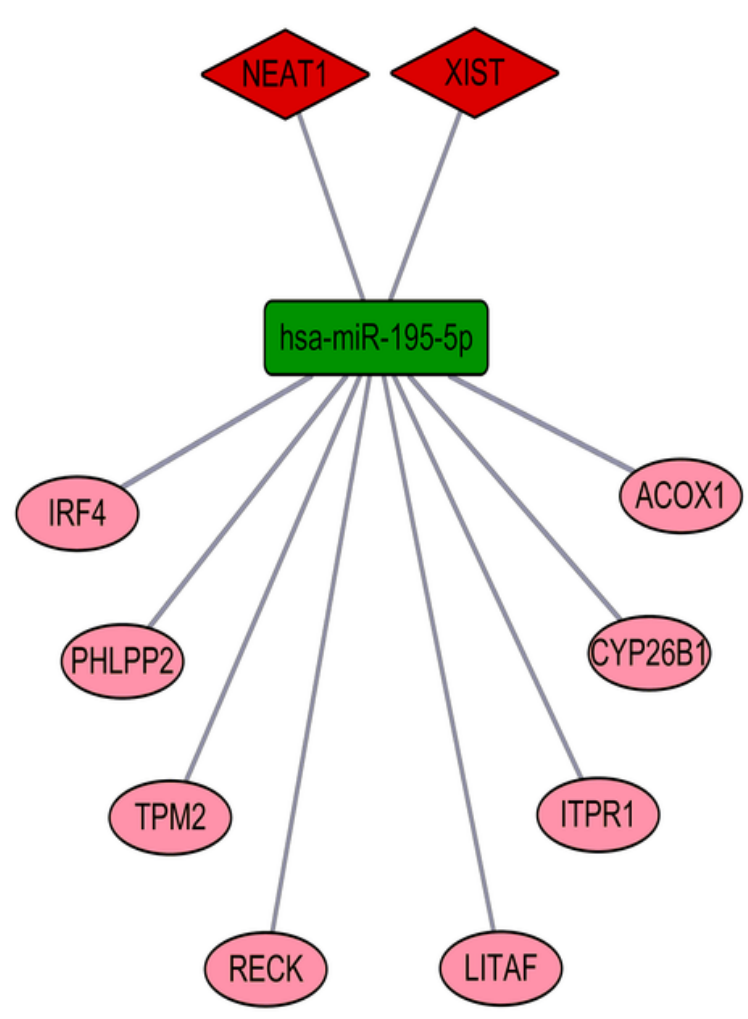

B
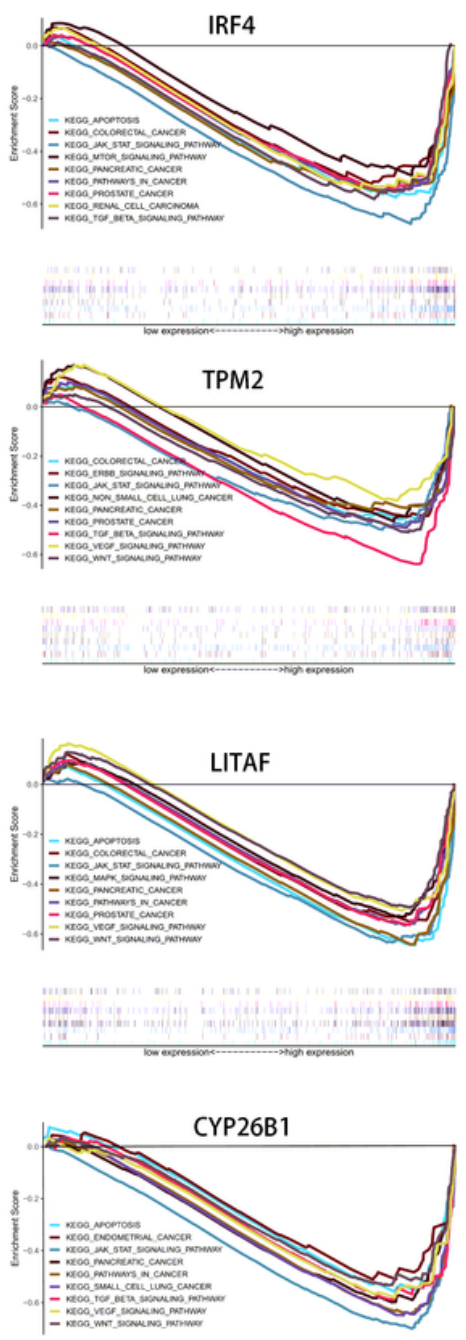

filong
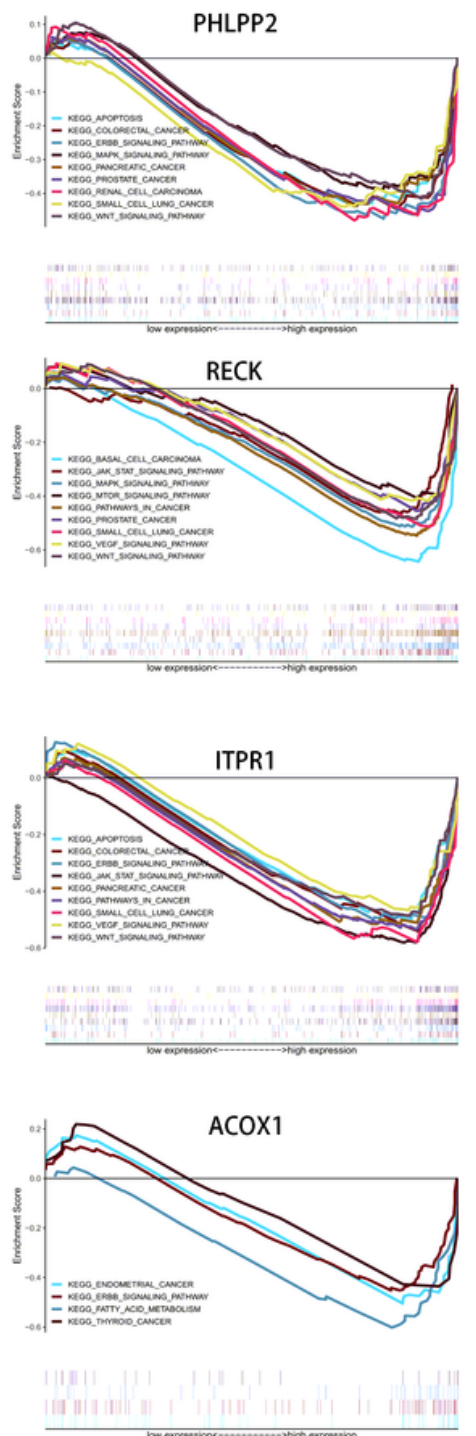

\section{Figure 5}

Construction of survival related IncRNA-miRNA-mRNA regulatory network in human CRC. (A) The ceRNA regulatory network associated with prognosis of patients with CRC. The red diamond represents key IncRNA. The green round rectangle represents key miRNA. The pink ellipse represents key mRNA. (B) GSEA enrichment analysis of mRNAs in the ceRNA regulatory network. 
A

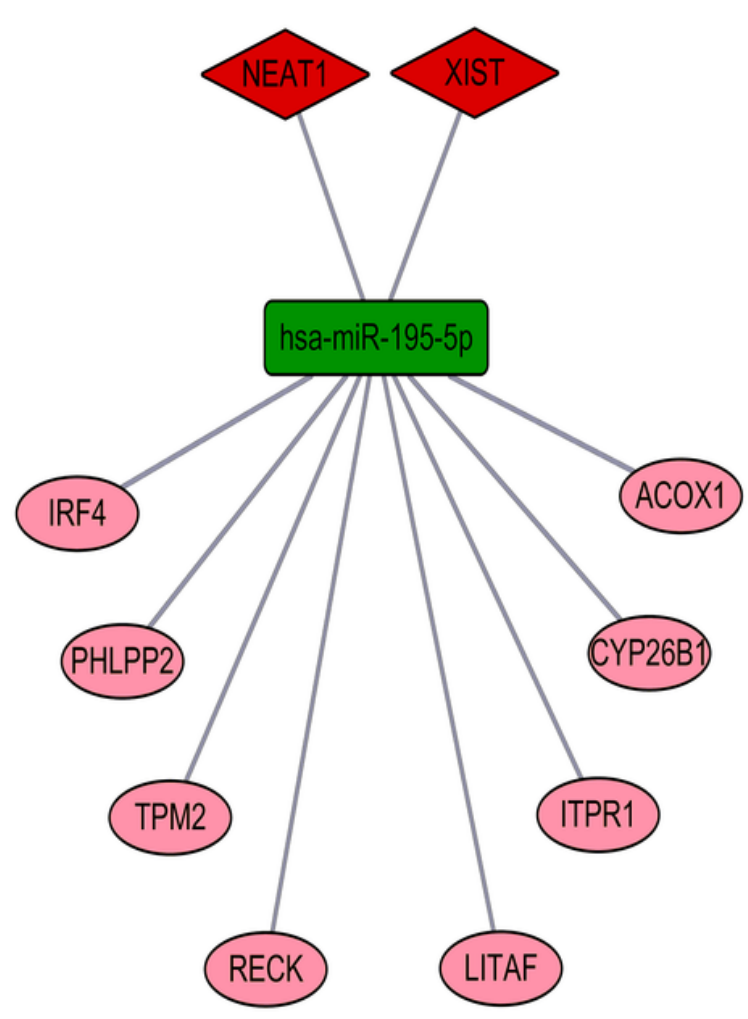

B
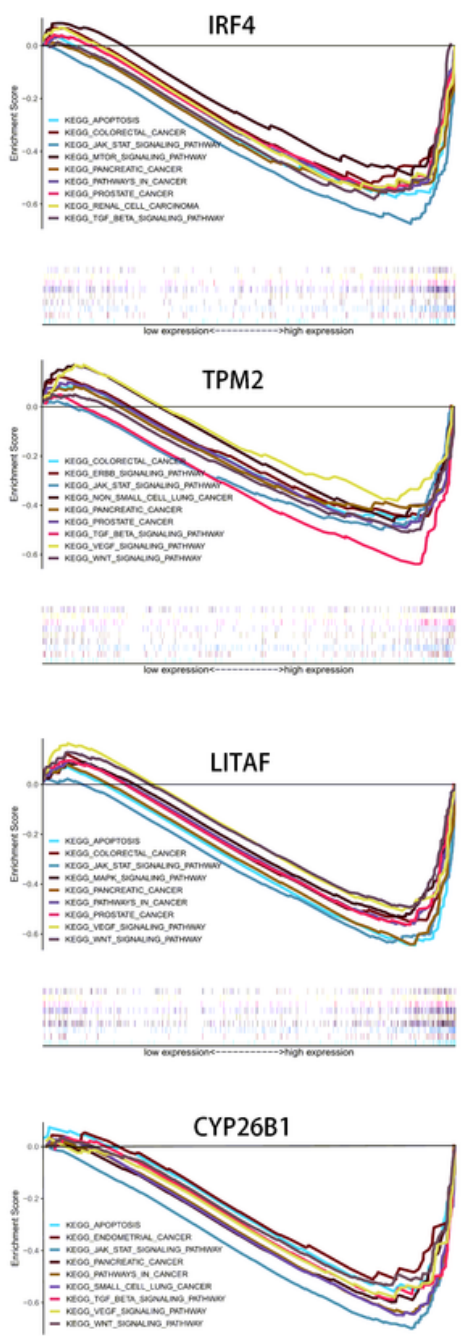

filong
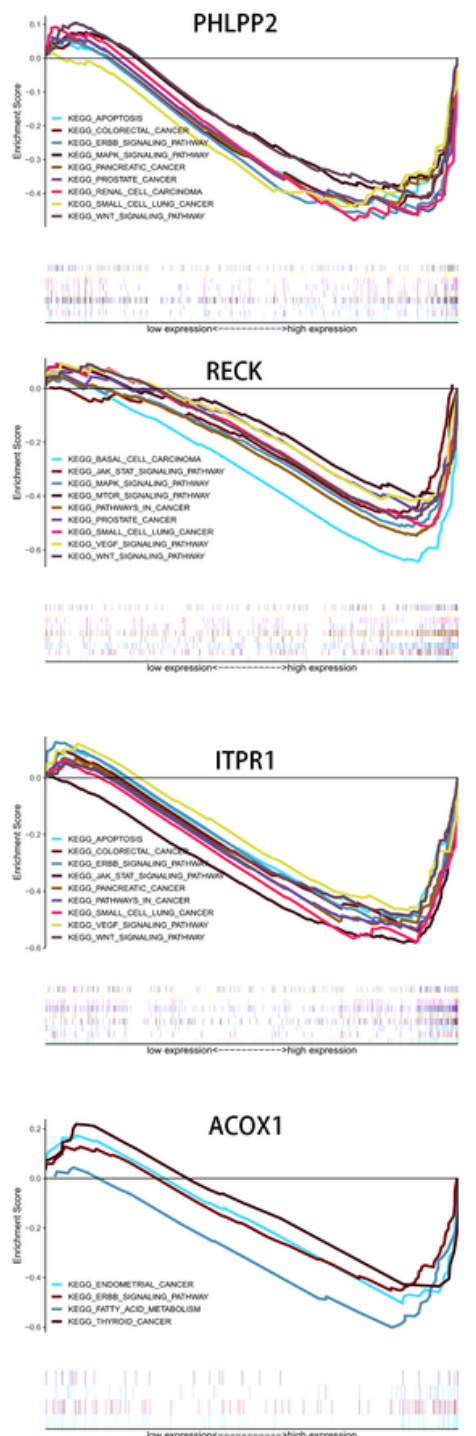

\section{Figure 5}

Construction of survival related IncRNA-miRNA-mRNA regulatory network in human CRC. (A) The ceRNA regulatory network associated with prognosis of patients with CRC. The red diamond represents key IncRNA. The green round rectangle represents key miRNA. The pink ellipse represents key mRNA. (B) GSEA enrichment analysis of mRNAs in the ceRNA regulatory network. 
A

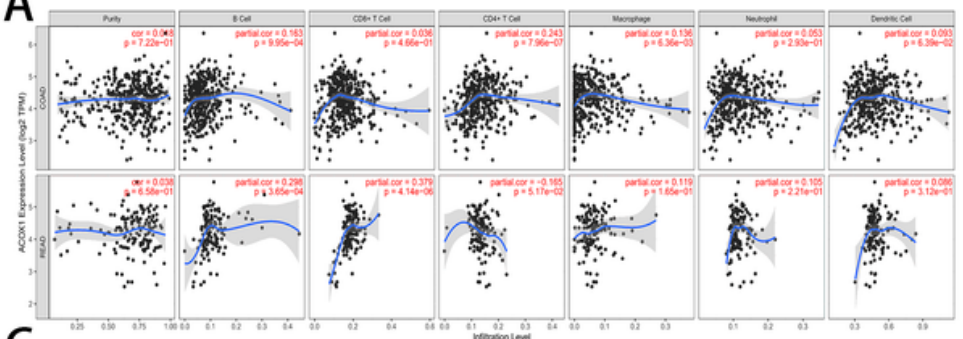

C

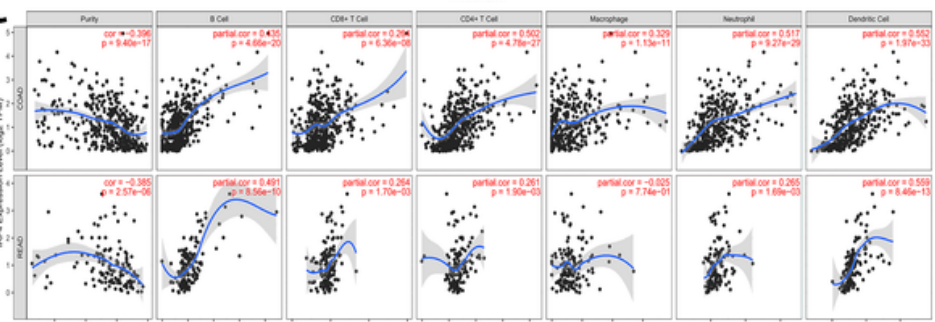

$\mathrm{E}$

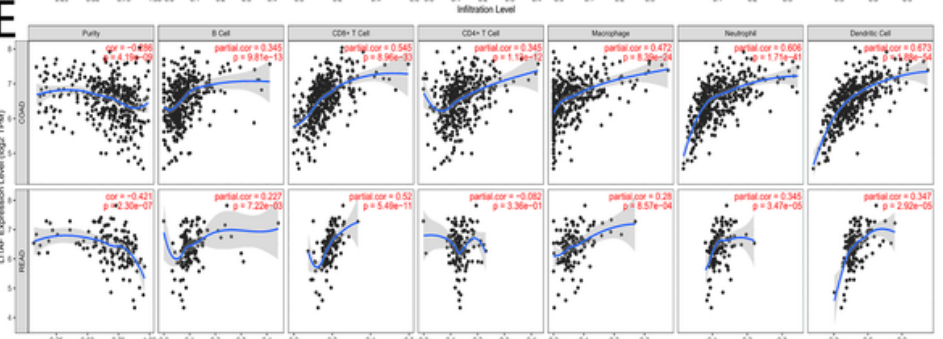

G

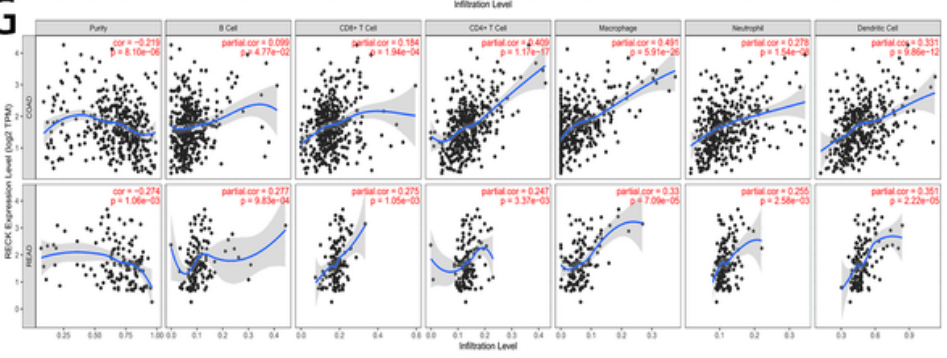

B

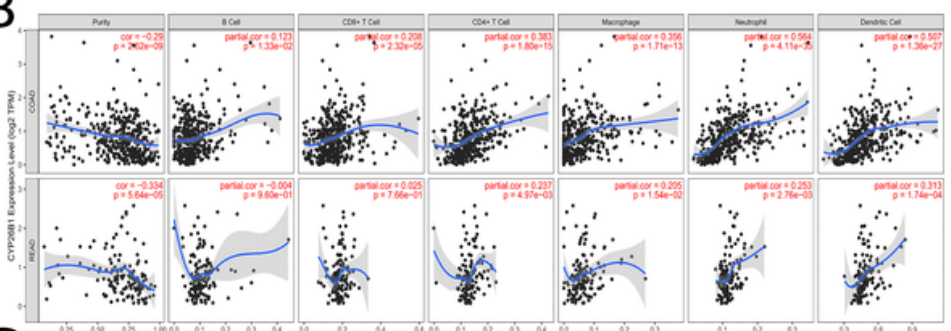

D
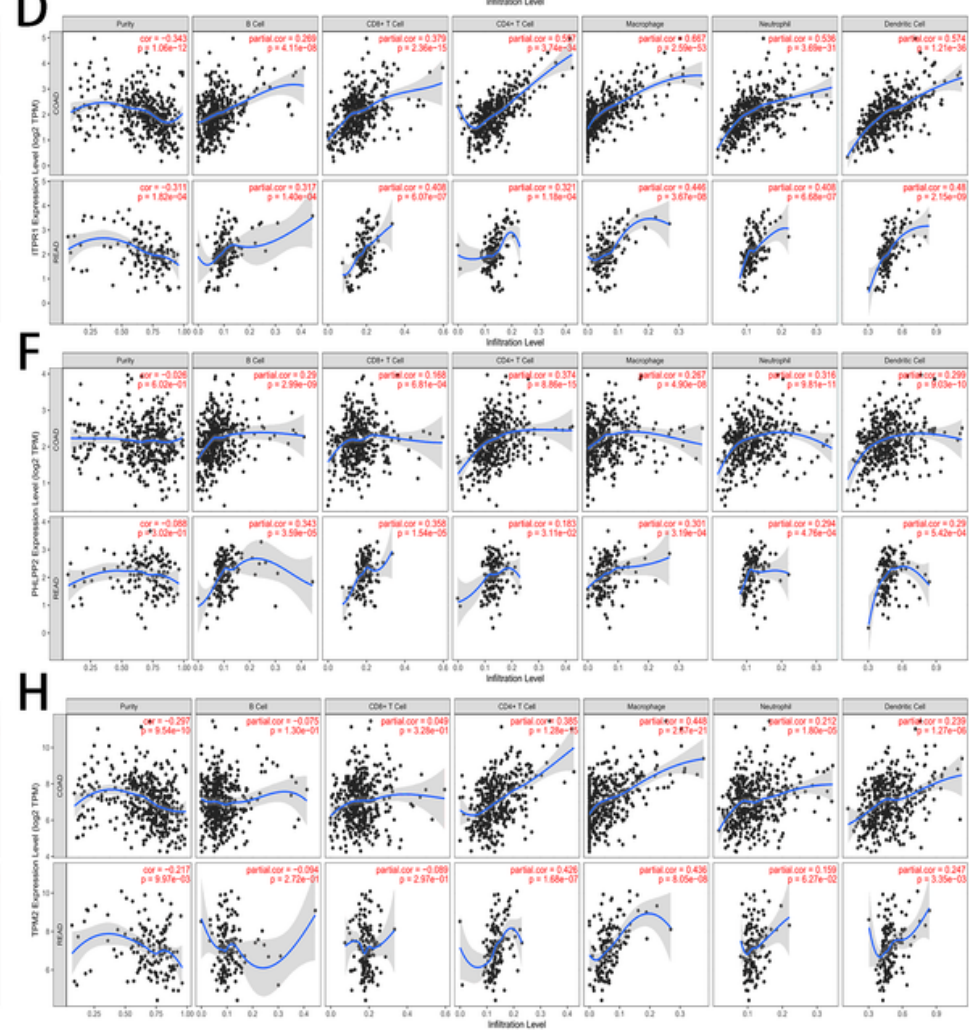

Figure 6

Association of ceRNA network expression with immune infiltration level in CRC. (A-H) Association of mRNA expression in the survival related ceRNA regulatory network with immune infiltration level in CRC. 
A

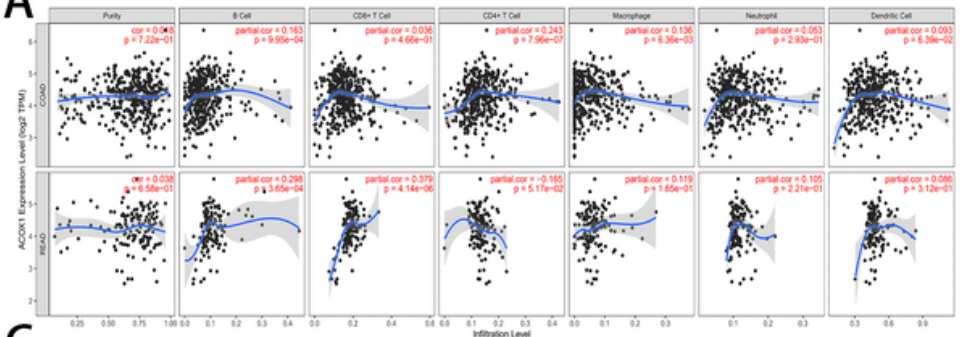

$\mathrm{C}$

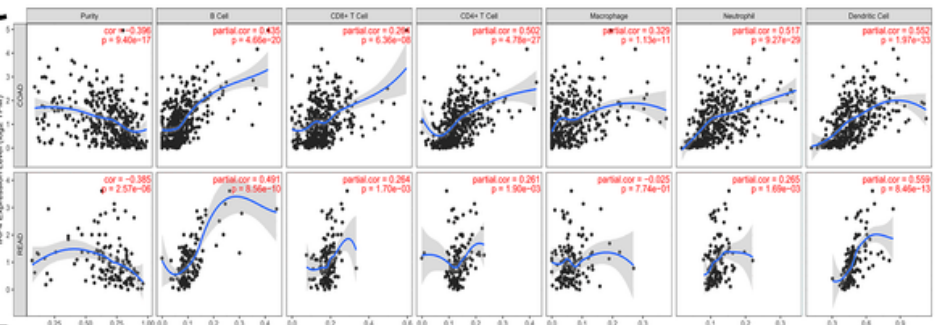

$\mathrm{E}$

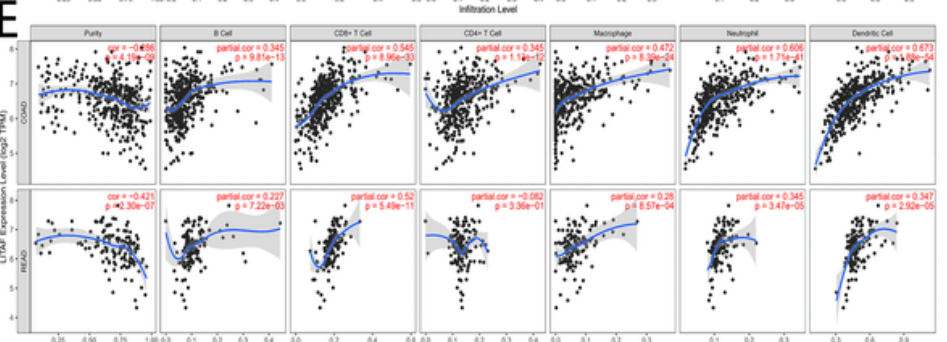

G

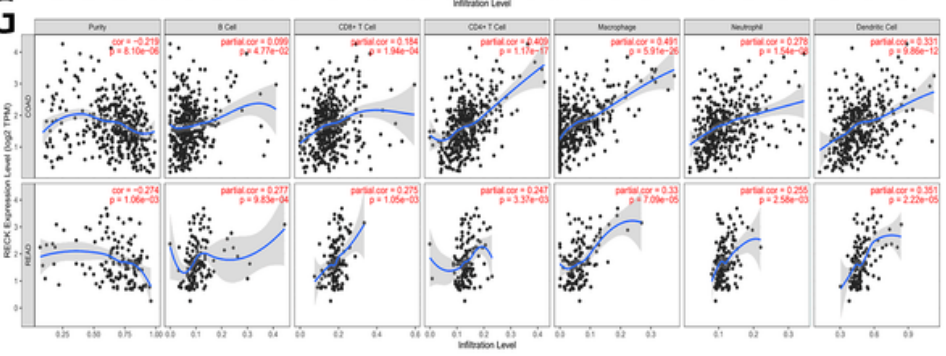

B

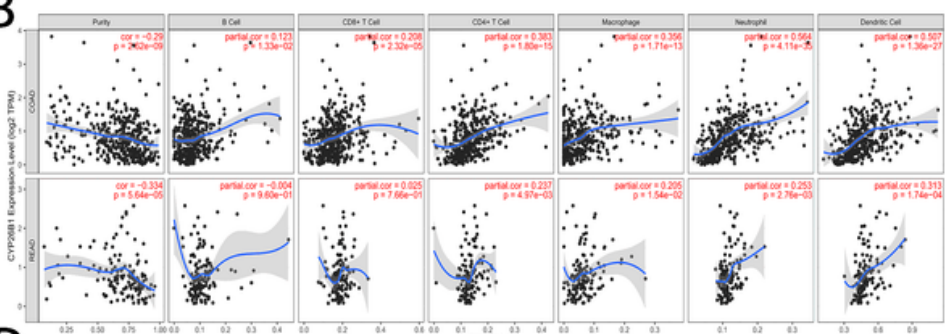

D
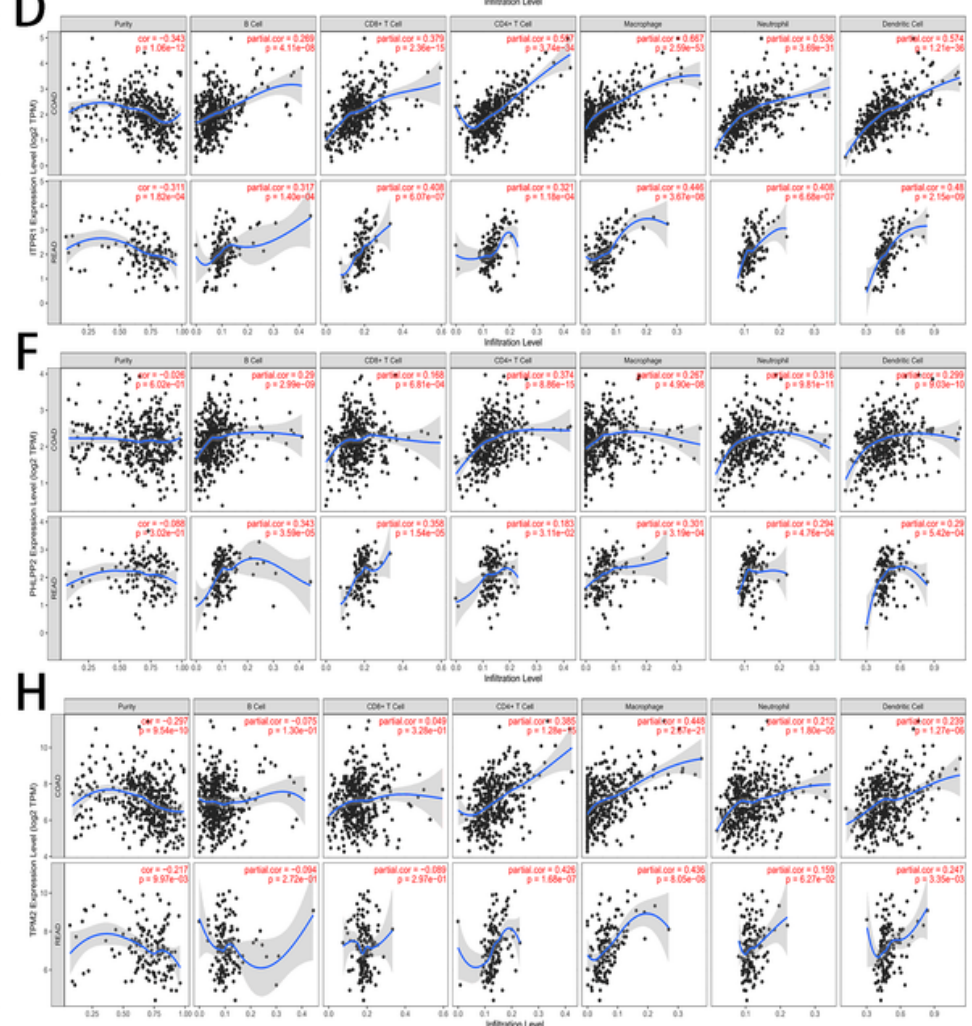

Figure 6

Association of ceRNA network expression with immune infiltration level in CRC. (A-H) Association of mRNA expression in the survival related ceRNA regulatory network with immune infiltration level in CRC. 

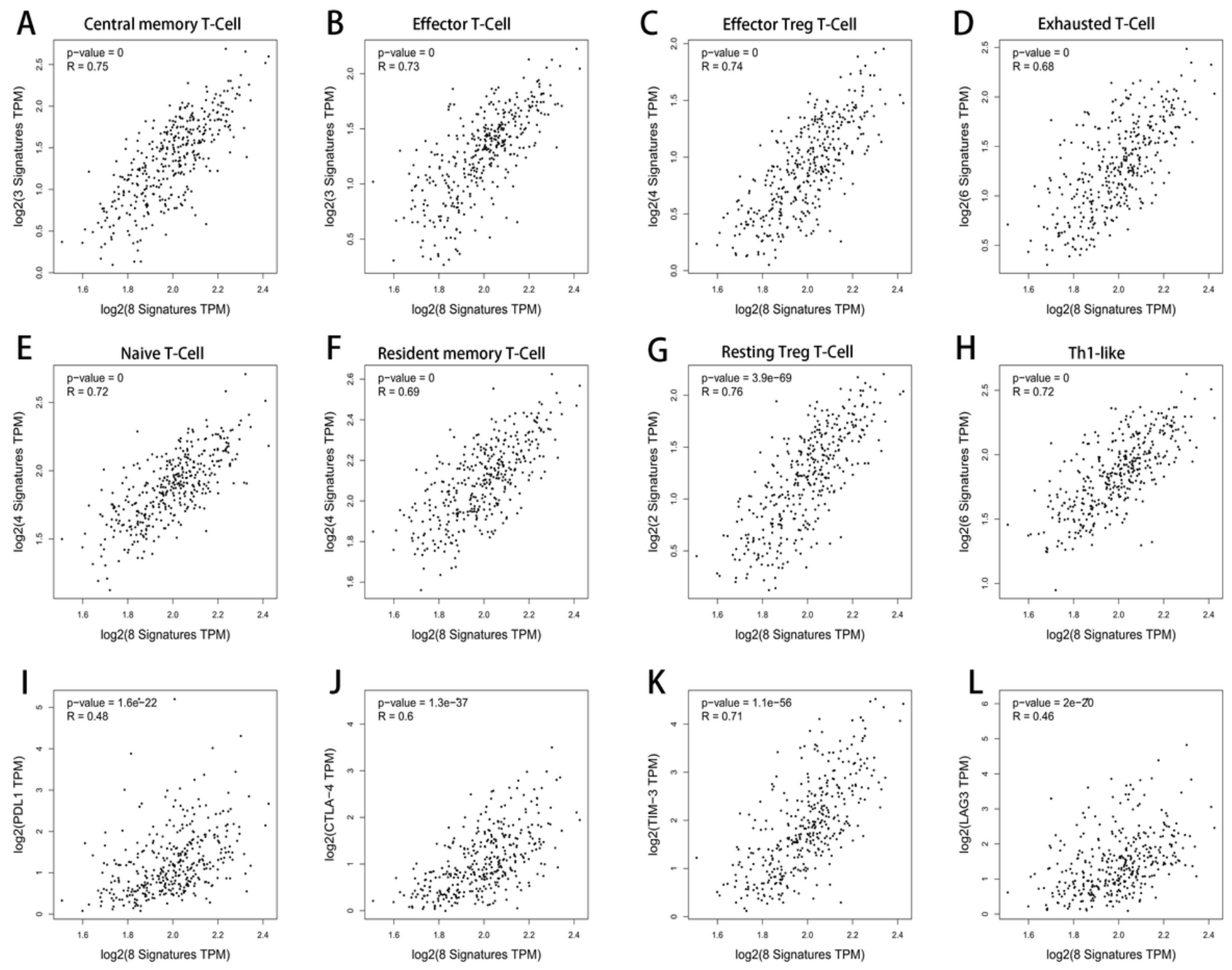

\section{Figure 7}

Association of ceRNA network expression with T cell immune infiltration abundance and immune checkpoint expression level in CRC. (A-H) Expression of mRNAs in the survival related ceRNA network was significantly correlated with immune infiltration abundance of T cells. (I-L) Expression of mRNAs in the survival related ceRNA network was significantly correlated with the expression level of immune checkpoint. 

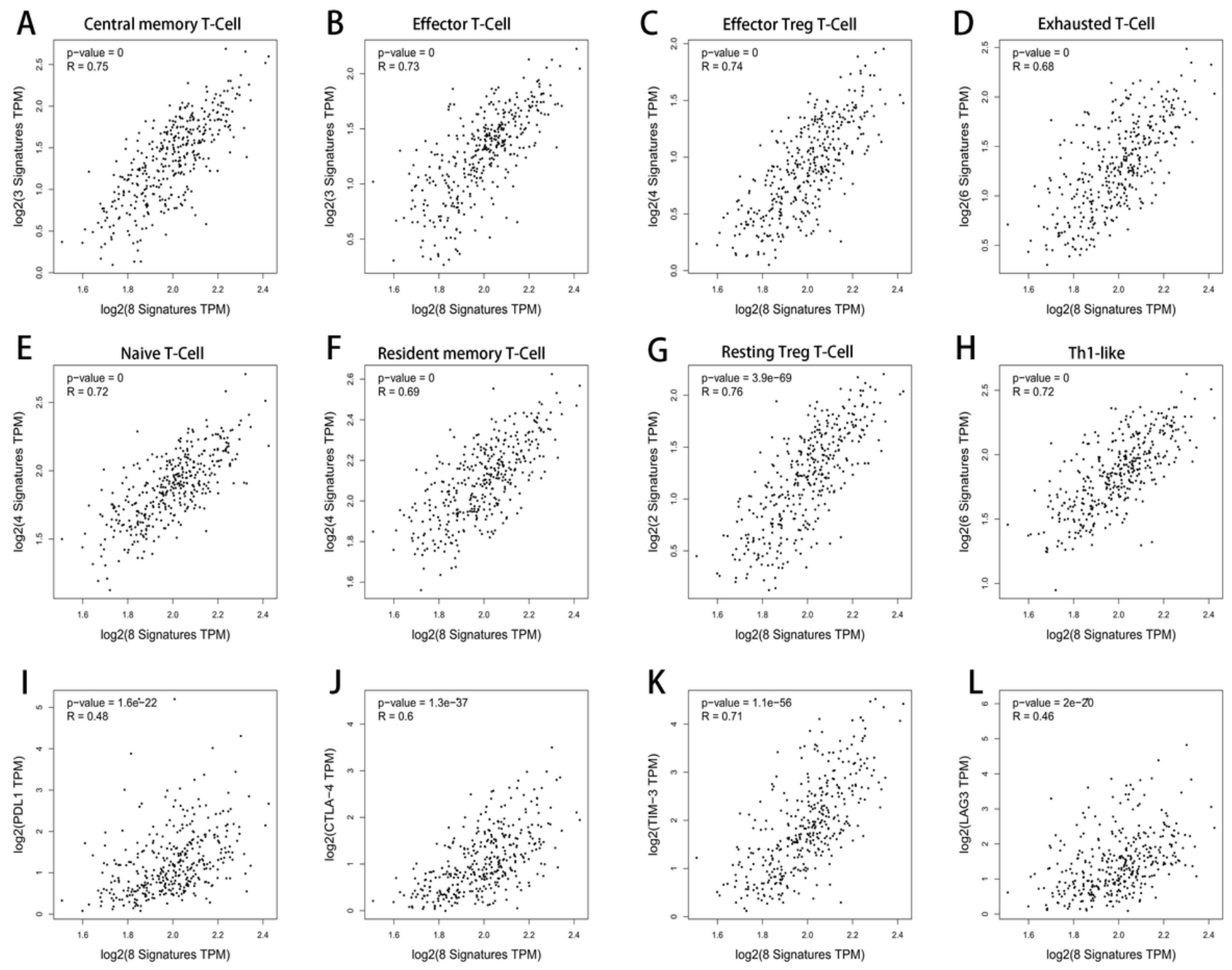

\section{Figure 7}

Association of ceRNA network expression with T cell immune infiltration abundance and immune checkpoint expression level in CRC. (A-H) Expression of mRNAs in the survival related ceRNA network was significantly correlated with immune infiltration abundance of T cells. (I-L) Expression of mRNAs in the survival related ceRNA network was significantly correlated with the expression level of immune checkpoint. 